\title{
Virtual Antenna Array for Minimization of DOA Estimation Systematic Error Caused by Scattering of Incident Waves on Antenna Carrier Body
}

\author{
Yuri G. Pasternak ${ }^{1}$, Aleksander V. Ashikhmin ${ }^{2}$, Yuri A. Rembovsky ${ }^{2}$, Sergey M. Fedorov ${ }^{1, * \mathbb{C}}$ \\ and Dmitry V. Zhuravlev ${ }^{1}$ \\ 1 Faculty of Radio Engineering and Electronics, Voronezh State Technical University, Voronezh 394006, Russia; \\ pasternakyg@mail.ru (Y.G.P.); ddom1@yandex.ru (D.V.Z.) \\ 2 IRCOS JSC, Moscow 129626, Russia; ashihminav@ircoc.vrn.ru (A.V.A.); yu.rembovsky@ircos.ru (Y.A.R.) \\ * Correspondence: fedorov_sm@mail.ru
}

Received: 6 January 2020; Accepted: 2 February 2020; Published: 10 February 2020

\begin{abstract}
The Direction of Arrival (DOA) estimations of systematic errors are caused by diffraction distortions of the measured spatial structure of a electromagnetic field. These distortions result from scattering of incident waves on the antenna system and nearby scatterers (mobile carrier body, antenna mast, underlying surface, etc.) in wide frequency band, including the resonant frequencies of nearby objects. This article proposes a method for minimizing the DOA estimation systematic error by forming an additional virtual receiving channel—a Virtual Antenna Array (VAA). The VAAs were formed by use of classical apparatus of electrodynamics-the Huygens-Kirchhoff principle, the method of equivalent fields and sources, and the quasistatic approximation of the field based on the theory of analytical functions of the complex variable (Cauchy integral, Laurent series). The proposed method does not require calibration of the antenna system or a priori information about the geometry and material properties of the scatterers (dry or wet soil, opened or closed vehicle doors, etc.). Therefore, it gives good results in cases of mobile and stationary arrays, or changing carrier body geometry.
\end{abstract}

Keywords: DOA estimation accuracy; virtual antenna array; direction finding; wave scattering; spatial field distribution; approximation methods

\section{Introduction}

The term "Virtual Antenna Array" (VAA) denotes a set of spatial samples of electric or magnetic field components approximated in the vicinity of the elements of a Real Antenna Array (RAA) and nearby scatterers-the carrier body, underlying surface, and other objects. Studies from the authors of [1-14] are devoted to VAA methods (also known as "interpolated array" or "phantom array"), and based on two main approaches: (1) full-scale measurement (or calculation) of radiation patterns of the RAA elements, taking into account the influence of the carrier body and underlying surface $[2,5-9,11]$; (2) interpolation of the field at points between the RAA elements [1,10]. Use of VAA methods increases the resolution of the equipment and an accuracy of Direction of Arrival (DOA) estimation for radio [1-13] or acoustic sources [14].

However, these approaches have certain disadvantages: the procedures for measuring (or calculating) the complex vector radiation patterns of RAA elements are labor-intensive, considering the scattering of waves on the carrier body and underlying surface; the frequency overlap coefficient of modern direction finders is more than 1000; the conductivity of the underlying surface can vary dozens of times; the geometry and material properties of the carrier body can vary significantly during 
operation (the scattering properties of the carrier body are changed with open or closed doors or roof hatches; contacts between the roof and the carrier body can change due to corrosion).

Friedlander [1] proposed an approach to apply the ROOT-MUSIC method, which was initially applicable only for linear equidistant antenna arrays, to arrays of arbitrary configuration. This approach is based on partition of the field observed by antenna into sectors (a $360^{\circ}$ wide sector is of particular interest) in which we search (by the least squares method) the transformation matrix of the measured phase relationships of the original antenna and VAA.

Additionally, Friedlander [1] studied a modification of the ROOT-MUSIC method based on the use of the signal subspace of VAA instead of the spatial correlation matrix, and the procedure for selecting the polynomial roots corresponding to the true DOA. This algorithm can be applied to resolve correlated signals.

The authors of [2] studied a method of forming a VAA with dimensions approximately two times larger than those of RAA. This method is based on use of received signal statistical cumulants with an order of more than two, characterized by very weak resistance to measurement errors of received signals parameters and has not yet found practical application.

Pesavento et al. [3] proposed performing an interpolation between RAA and VAA as a minimization problem with multiple constraints in the form of inequalities. Their approach is based on minimizing the interpolation error in the sector, and many spatial filters in the rest of the space. This allows the processing to be stable to signals arriving not from the interpolation sector.

Buhren et al. [4] proposed an interpolation method based on the formation of VAA as a shifted copy of RAA. Thus, we do not need to choose the number of VAA elements, interelement distance, or array orientation. The created invariance to the shift can be used in the ESPRIT method. This method also requires sector-by-sector processing.

Hyberg [5] proposed a method of creating a transformation matrix based on a decrease in the bias of the DOA estimates. Taking into account the terms of the second order of expanding the output function of the MUSIC method in a Taylor series, he obtained a formula for DOA error, and used it to synthesize a transformation algorithm with a reduced error based on the least squares method. Similar to the Friedlander approach [1], signals outside the transformation sector were ignored. Additionally, Hyberg expanded his approach to take into account the finite time of observation.

Belloni et al. [6] performed an analysis of DOA errors of transformation into the beamspace, and obtained an expression for DOA error. Using this expression, a procedure was synthesized to reduce the variance of estimates caused by the application of the transformation into the beamspace. This procedure was used to formulate a criterion for choosing the number of VAA elements. The criterion follows from the analysis of the IFFT (inverse fast Fourier transformation) of the array impulse response, and it is optimal for maximizing the aperture of the VAA with restrictions on the number of virtual elements. By use of this criterion, we can obtain standard deviations of estimates close to the value of the Cramer-Rao boundary.

Buhren et al. [8] proposed and studied a method of formation of VAA named the manifold separation technique. This method provides accurate interpolation, but requires a fairly dense grid of measured steering vectors, and their values must be determined with high accuracy.

Field measurements from the reference generator are noisy, which additionally reduces the quality of the transformations. The relationship between the noise level and the number of modes required for interpolation was established by authors of [8]. Additionally, they obtained expressions that describe DOA errors caused by calibration noise and transformation.

Chandran [9] studied the modal space processing, and showed that by use of Jacobi-Anger expansion of function $\exp \left(-i k_{0} x \cos (\theta)\right)$, which describes the propagation of a plane electromagnetic wave at an angle $\theta$ to the coordinate axis $x$, it is possible to form a VAA.

Pieter van Rooyen et al. [10] formed a VAA by two-dimensional (along the $x$ and $y$ axes) Lagrange interpolation. There is a similar approach differed only by use of Kotelnikov series for interpolation. 
Additionally, there is method based on substitution of scatterers of complex shape by simple scatterers; in particular, spheres [12] or cylinders. However, this method requires the coordinates of scatterers' centers. The limitations of such an approach are obvious in the case of objects of very complex shapes; for example, those with resonant holes.

Xiaopu Wang et al. [14] proposed a method based on imitation of virtual uniform antenna array by rotating a smartphone around the user's body for $360^{\circ}$. This method can emulate the sensitivity and functionality of a uniform antenna array to estimate the angle of arrival of the target signal, and does not require any additional specialized devices. Nguyen et al. [15] studied a similar approach based on large-scale virtual array analysis of the spatially sampled microwave channel. Data for formation of VAA were obtained by an omnidirectional antenna continuously moving during the measurement. Such approaches are simple, but you need to move the receiving device during measurement, which leads to increases in time costs and DOA estimation error.

Chengwei Zhou et al. [16] studied the coarray interpolation-based DOA estimation algorithm via covariance matrix reconstruction. The proposed approach can be used to generate a uniform linear array in the coarray domain of coprime array, such that all the signals of the non-uniform coarray can be utilized. This allows us to apply DOA estimation methods designed for the uniform linear array to coprime array which coarray is non-uniform. Min Lin et al. [17] proposed the VA-MUSIC algorithm based on use of complex conjugate counterpart of the array output, which introduces a virtual array to extend the equivalent array aperture, so that it can handle more sources than sensors. Based on dividing the virtual array into many subarrays, and forming a new array covariance matrix, the proposed algorithm is suitable for estimating both the uncorrelated and correlated signal sources. These approaches [16-20], based on interpolation of the field at points lying between the antenna array elements, do not increase the dimensions (therefore, do not reduce an influence of the distortions caused by nearby scatterers) of the VAA more than that of the RAA.

The aim of this article is to achieve a significant increase of DOA estimation accuracy by the use of the VAA with dimensions up to 2-3 times larger than that of the RAA, without using any information (such as geometry, material properties, and characteristics of the scattering by nearby objects) about the carrier body of RAA or underlying surface.

The remainder of this paper is organized as follows. In Section 2, we introduce the methods of formation of VAA based on auxiliary field sources and theory of analytic functions of a complex variable. Simulation and measurement results are provided in Section 3. In this section, we study different variations of proposed methods under various conditions. We conclude this paper in Section 4.

\section{Formation of Virtual Antenna Array}

The input data for the formation of VAA are the signal complex amplitudes $u_{1}^{r}, u_{2}^{r}, \ldots, u_{M}^{r}$ at outputs of RAA elements, measured at a certain frequency $f_{0}$. The output data are estimates of the signal complex amplitudes $u_{1}^{v}, u_{2}^{v}, \ldots, u_{M}^{v}$ at outputs of VAA elements located on a circle of radius $r_{v}$ greater than radius of the RAA $r_{r}$.

Electrically short symmetrical vibrators, loaded with high-frequency amplifiers, were used as elements of circular RAA. All vibrators were oriented along the vertical axis $z$. With their help, we obtained the estimates of complex amplitude of $E_{z}$-field at the corresponding points, and used them as the nodal values for the approximation procedure of scalar field $E_{z}(x, y)$ at locations of phase centers of VAA elements.

Formation of VAA by approximation of the field, measured at the set of points, is possible because of the Huygens-Kirchhoff principle, whose mathematical description comprises the Lorentz lemma (in the vector case) and the Kirchhoff integral (in the scalar case).

The amplitude-phase distribution of the field can be measured by RAA consisting of electrically short vibrations. This amplitude-phase distribution is, to a certain extent, a holographic image of the nearby scatterers (the antenna system itself, its carrier body, underlying surface, etc.). The problem is how to extract information about the structure of the scattered field if we know only one of its 
scalar components in a small number of points-phase centers of the electrically small elements of RAA, which are actually capacitive sensors of electric field. In this article, we study two methods for extracting information about electromagnetic field from signals at the elements of antenna system: (1) a method based on the basic principles of electrodynamics [21] (Huygens-Kirchhoff principle, Lorentz lemma, Kirchhoff integral, equivalent fields principle, and equivalent currents principle); (2) a method based on the theory of analytic functions of a complex variable [22] (Cauchy integral, Poisson integral, and Laurent series), which can be used because the scattered field can be analyzed in the quasistatic approximation.

\subsection{Method Based on Auxiliary Field Sources}

It is possible to significantly (up to 50 times) reduce the order of a system of linear algebraic equations, those solution is complex amplitudes of the auxiliary field sources [23]. This can be achieved by transition to a finite number of auxiliary field sources located on closed integration surface $S_{u}$, inside which the surface of analyzed object $S_{o}$ is located. The ratio of dimensions of surfaces $S_{u}$ and $S_{o}$ should be within 1.5-2.

To form a VAA based on the Kirchhoff integral [21], we chose the method of auxiliary field sources [23] as the basic method for representing the field. We need to obtain the field values at points located on the same plane as the phase centers of symmetric electric vibrators of RAA, and so we use the scalar Green functions of linear sources as the basis functions for representing the field:

$$
G(P, Q)=-\frac{\pi \cdot i}{2} \cdot H_{0}^{1}\left(k_{0} r_{P, Q}\right),
$$

where $H_{0}^{1}(z)$ is Hankel function of the first kind of zero order; $i=\sqrt{-1} ; k_{0}$ is wave number; $r_{P, Q}$ is the distance between the observation point $P$ and the source point $Q$.

Formation of VAA consists of solving the following system of linear algebraic equations with respect to unknown complex amplitudes $U_{k}$ of auxiliary linear sources:

$$
\sum_{k=1}^{N} U_{k} \cdot G\left(P_{n}, Q_{k}\right)=u_{\text {real }}\left(P_{n}\right), n=1,2, \ldots, N,
$$

where $u_{\text {real }}\left(P_{n}\right)=u_{n}^{r}$ is the complex amplitude of the signal at output of n-th element of RAA consisted of $N$ elements.

Then, the signals at outputs of VAA (i.e., the estimates of $E_{z}$-field at points $p_{m}$ ) are calculated as follows:

$$
u_{\text {virtual }}\left(p_{m}\right)=u_{m}^{v}=\sum_{k=1}^{N} U_{k} \cdot G\left(p_{m}, Q_{k}\right), m=1,2, \ldots, M .
$$

The error in measurement of complex signal amplitudes $u_{n}^{r}$ and its influence on accuracy of the field approximation can be reduced by use the number of auxiliary field sources $K$ less than $N$. In this case, we can find $U_{k}$ and minimize the residual by the least squares criterion [22,24] as follows:

$$
\vec{U}=\left[U_{1}, \ldots, U_{K}\right]^{T}=\left(\left[G_{n, k}\right]^{H} \cdot\left[G_{n, k}\right]\right)^{-1} \cdot\left[G_{n, k}\right]^{H} \cdot\left[u_{1}^{r}, \ldots, u_{N}^{r}\right]^{T},
$$

where $\vec{U}=\left[U_{1}, \ldots, U_{K}\right]^{T}$ is a vector consisting of complex amplitudes $U_{K}$ of auxiliary linear sources; $G_{n, k}=G\left(P_{n}, Q_{k}\right)$ are the elements of matrix with $N$ rows and $K$ columns; $H$ is a sign of Hermitian transposition; $\left[u_{1}^{r}, \ldots, u_{N}^{r}\right]^{T}$ is a vector with elements representing a complex amplitude of the signals at the outputs of the elements of RAA. 


\subsection{Method Based on the Theory of Analytic Functions of a Complex Variable}

When the diameter of RAA is shorter than the wavelength $\left(2 r_{r}<\lambda_{0}\right)$, we can form a VAA by another method, based on measured values of the fields and their analytical continuation using the theory of analytical functions of a complex variable $z=x+i y$; in particular, the Cauchy integral $[25,26]$ or Laurent series [27]. This method is applicable because we can use a quasistatic description of the field in an electrically small region: if $k_{0}^{2}=\left(2 \pi / \lambda_{0}\right)^{2} \ll 1$, the Helmholtz equation can be replaced by the Laplace equation, whose solutions are harmonic functions. Furthermore, any analytical function of a complex variable can be represented as the sum of a harmonic function and a harmonically conjugate function [22]. Therefore, the analytic functions of a complex variable can be used for the quasistatic approximation of the field.

The $E_{z}$-field over the contour of equidistant circular RAA with radius $r_{r}$ can be described, based on the measured field values $u_{1}^{r}, u_{2}^{r}, \ldots, u_{N}^{r}$, by the following polynomial:

$$
U_{A A}\left(z=r_{r} \cdot \exp [i \phi]\right)=\sum_{n=1}^{N} B_{n} \cdot \exp \left[\frac{i(n-1) \phi}{N}\right],
$$

where we find $B_{n}$ coefficients by the least squares method [22,24].

Using the Poisson integral (a special case of the Cauchy integral for a contour in the form of a circle), we can write an integral equation of the first kind, which connects the fields over the contours of RAA and VAA:

$$
\begin{aligned}
& U_{A A}\left(z=r_{r} \cdot \exp [i \phi]\right)=\frac{1}{2 \pi} \int_{0}^{2 \cdot \pi} U_{V A A}\left(r_{v} \cdot \exp [i \psi]\right) \frac{r_{v}^{2}-r_{r}^{2}}{r_{v}^{2}-2 r_{v} r_{r} \cdot \cos (\psi-\phi)+r_{r}^{2}} d \psi= \\
& =\frac{1}{2 \pi} \int_{0}^{2 \cdot \pi}\left\{\sum_{k=1}^{K} X_{k} \cdot \exp \left[i(k-1) \cdot \frac{\psi}{K}\right]\right\} \frac{r_{v}^{2}-r_{r}^{2}}{r_{v}^{2}-2 r_{v} r_{r} \cdot \cos (\psi-\phi)+r_{r}^{2}} d \psi .
\end{aligned}
$$

Equation (6) can be solved with respect to unknown coefficients $X_{k}$ by the collocation method, with the limited spoilage method [22] used as a regularization method.

Another method of field approximation is based on the expansion of function $U_{A A}(z)$ in a narrow ring of width $\delta\left(r_{r}-\delta / 2 \leq r \leq r_{r}+\delta / 2\right.$, where $\left.\delta \ll r_{r}, \delta \ll \lambda_{0}\right)$, inside of which the phase centers of the RAA elements are located, in a Laurent series [27]:

$$
U_{A A}(z)=\sum_{k=-\infty}^{\infty} c_{k} z^{k}
$$

where $c_{k}=\frac{1}{2 \pi i} \int_{G} \frac{U(\zeta) d \zeta}{\zeta^{k+1}}$.

We can use Equation (7) to find the derivative functions $U_{A A}(z)$ in the radial directions $\delta U(\phi, r) / \delta r$, $\delta^{2} U(\phi, r) / \delta r^{2}$, etc., and estimate the function $U_{A A}\left(\phi, r_{v}\right)$ values on a circle of radius $r_{v}=r_{r}+L$ by use of segment of the Taylor series (at a considerable distance from the contour of RAA: $L \gg \delta$, where approximation (7) is no longer applicable due to the fact that by it we describe the field only in a narrow circle of width $\delta$ ):

$$
U\left(\phi, r_{v}\right) \approx U\left(\phi, r_{r}\right)+L \cdot \frac{\delta U(\phi, r)}{\delta r}+0.5 \cdot L^{2} \cdot \frac{\delta^{2} U(\phi, r)}{\delta r^{2}}+\ldots
$$

\subsection{Some Properties of the Presented Methods}

There are advantages and disadvantages of the presented methods for forming a VAA. Their common advantage is that the formed VAA can increase (by 2-3, or more times) the accuracy of DOA estimation in the resonance frequency range of wave scattering on a RAA, its carrier body, the underlying surface, and other nearby objects [28]; they do not require any information about the 
geometry and material properties of nearby objects, or their characteristics of wave scattering, which can be seen from Equations (1)-(8).

As example, we researched an influence of the presence (or absence) of a rood hatch of $680 \times 350 \mathrm{~mm}^{2}$ in the roof of the carrier body (GAZelle minibus) of a direction finder antenna array. The roof hatch was located directly below the antenna system with a radius of $500 \mathrm{~mm}$, offset by $1.5 \mathrm{~m}$ towards the rear of the vehicle. Study showed that in all these cases the efficiency of the bearing correction by VAA method remained acceptable (the root-mean-square error of DOA estimation was reduced by $1.932,1.709$, and 1.948 times, respectively, for each case; the study was conducted in frequency range $25-150 \mathrm{MHz}$ ). The maximum bearing error decreased by four or more times. The analyzed antenna system consisted of nine symmetric electric vibrators $160 \mathrm{~mm}$ long and $20 \mathrm{~mm}$ in diameter, and loaded on high-frequency amplifiers with an average input resistance of about $1000 \mathrm{ohms}$.

Numerical implementation is the simplest for the VAA method based on the Cauchy integral (Equations (5) and (6)) and Laurent series (Equations (7) and (8)). In the first case (Equations (5) and (6)), the problem reduces to solving a system of linear algebraic equations, whose order is limited by the number of RAA elements (on mobile direction finders, we can rarely see a circular array with more than nine elements [29]). In the second case (Equations (7) and (8)), the computational cost is mainly in finding the coefficients of the Laurent series (Equation (7)). However, the simplicity of these methods and their low computational cost lead to reduction of radius of the formed VAA and accuracy of the field approximation, compared to the method of auxiliary field sources (1-4). The limitations of methods which are based on field approximation by the theory of analytic functions of a complex variable were caused by the quasistatic approximation of the problem.

Additionally, we can form a VAA by use of the method of auxiliary field sources (Equations (1)-(4)); the radius of VAA will reach a value of three radiuses of RAA. That will increase DOA estimation accuracy: the standard error will be reduced by 2-3 times; the maximum DOA estimation error caused by resonant scattering of waves by the antenna system, the carrier body, and the underlying surface will be reduced by four or more times. At the same time, a field measurement error made by RAA has less influence on field approximation due to use of the quasisolution procedure (Equation (4)).

The novelty of proposed approaches and methods for the VAA formation is due to the following circumstances.

In studies of other authors, VAAs are formed on the basis of the following premises. First, a real antenna system is transparent for electromagnetic waves; i.e., it does not distort the spatial structure of the observed electromagnetic field. The assumed transparency of a RAA is reflected in the methods described by other authors, particularly when they use expression for a plane electromagnetic wave in equations describing the structure of the observed field. In reality, the antenna array, mast, phone case, or carrier body (and in portable radio receiving systems-the user's body) can significantly distort the spatial and temporal structure of the field due to scattering and absorption of waves on objects.

Second, the scattering properties of the antenna system and the objects surrounding it are implicitly taken into account during the calibration, which consists of creating a database that stores complex signal amplitudes at the outputs of the antenna elements for different values of the angles of incidence and for different polarizations.

In the proposed methods, the spatial distribution of the observed field distorted by the antenna system and surrounding objects is described using the superposition of the fields created by the system of auxiliary field sources. We considered two options for the location of auxiliary field sources: without using a priori information about the geometry of the scatterers; using a priori information about the geometry of the scatterers, when it is known and does not change during operation. Thus, a key feature of our VAA methods is the possibility of VAA formation under conditions of a priori unknown geometry and material properties of the scatterers.

Another fundamental difference in the proposed VAA methods is that we do not use the calibration of a RAA. This process is very laborious and has to be repeated periodically during operation of the equipment due to changes in the scattering properties of objects surrounding the antenna system-the 
carrier body is exposed to atmospheric influences, electrical contacts between parts of the structure can change during operation, and the properties of the underlying surface can change significantly. Therefore, we have developed methods for the VAA formation that are invariant to the geometry and material properties of the surrounding scatterers. Moreover, in a number of applications (for example, in mobile phones) it is in principle impossible to calibrate the antenna system because the position of the antenna system relative to the user's body varies, and the geometric dimensions of users can vary widely.

Another proposed approach for the formation of VAA is to use the analytical functions of a complex variable, which are solutions of the Laplace equation; i.e., consideration of the quasistatic approximation of the observed spatial distribution of the field distorted by the scattering of waves on surrounding objects. This allows you to take into account the scattering properties of the carrier body, the antenna system, and its mounting elements without using a priori information about the geometry and material properties of the scatterers. This approach is applicable when the dimensions of the antenna system are significantly less than the wavelength.

Our analysis of publications of other authors did not reveal the above approaches to the VAA formation and methods for VAA implementation, which allows us to argue that the proposed approaches and methods of VAA forming are original. The advantage of our approaches and methods is that they do not need a priori knowledge of the geometry and material properties of the scatterers, they do not need calibration of the receiving antenna system, and they can provide significant increase in the accuracy of DOA estimating, along with a significant increase in resolution.

The aim of many recent studies is to increase the positioning accuracy of Global Navigation Satellite Systems (GNSS) by use of the Precise Point Positioning (PPP) technique. The authors of [30] compared a multi-GNSS PPP time and frequency transfer with different precise products. To improve multi-GNSS PPP time and frequency, they suggest employong a receiver clock offset model, considering the correlation of the receiver clock offsets between adjacent epochs using an a priori value. Kamil Maciuk [31] studied absolute positioning with the usage of PPP technique on different simulated sky view conditions. The data were processed in three modes: GPS-only, GLONASS-only, and hybrid GNSS (GPS+GLONASS) using three different elevation cut-off angles. Wang [32] studied a technique which uses a stand-alone GPS station to conduct millimeter-accuracy landslide monitoring; Rabbou et al. [33] studied a method of PPP accuracy enhancement using GPS/GLONASS observations in kinematic mode; Qiong Wu et al. [34] studied a dual-frequency PPP algorithm realized on a smartphone in static and kinematic modes. A method for extracting line-of-sight ionospheric observables from GPS data using PPP was studied in [35].

The VAA method proposed in this article can form the additional virtual antenna elements, and therefore, enhance performance of above-mentioned PPP technique and positioning accuracy overall.

The proposed VAA methods were compared with the correlation-interferometric method of DOA estimation. The essence of the correlation-interferometric method is finding the maximum cross-correlation coefficient between measured (by RAA or VAA) amplitude-phase distribution on the antenna elements and a reference distribution in the studied frequency range and over azimuthal angular coordinate range from 0 to 360 degrees. The reference amplitude-phase distribution was chosen to be an equi-amplitude distribution with a phase distribution corresponding to a plane electro-magnetic wave propagating in a homogeneous medium. The direction to a radio source was taken as an angular estimate that corresponded to the maximum value of the cross-correlation coefficient [29]. In the correlation-interferometric method we use voltages $\vec{U}=\left[U_{1}, U_{2}, U_{3}, \ldots, U_{N}\right]^{T}$ calculated during the simulation, known wavelength $\lambda_{0}$, and known coordinates $x_{n}$ and $y_{n}$ of phase centers of antenna array elements. DOA estimations correspond to the maximum modulus of the following function:

$$
D(\phi)=\left|\sum_{n=1}^{N} U_{n} \cdot \exp \left[-i \cdot \frac{2 \cdot \pi}{\lambda_{0}} \cdot\left(x_{n} \cdot \cos (\phi)+y_{n} \cdot \sin (\phi)\right)\right]\right|,
$$

where angle $\phi$ varies from 0 to $2 \pi$ radians. 


\section{Simulation and Measurement Results}

\subsection{Approximations of the Measured Field by Plane Waves of Fixed Auxiliary Sources}

During numerical simulation and refinement of the proposed methods, the carrier body and elements of the antenna array were represented as perfect conductors; to simulate a high-frequency amplifier, the elements of antenna array were loaded on 1000-ohm resistors. The antenna array consists of nine symmetrical electric vibrators $160 \mathrm{~mm}$ long and $20 \mathrm{~mm}$ in diameter, which are located on a circle with radius of $500 \mathrm{~mm}$. The gap between the vibrators and roof of the carrier body was $30 \mathrm{~mm}$ (Figure 1). The mobile radio direction finding system of the "Article" family was used for full-scale experimental studies; Figure 2.

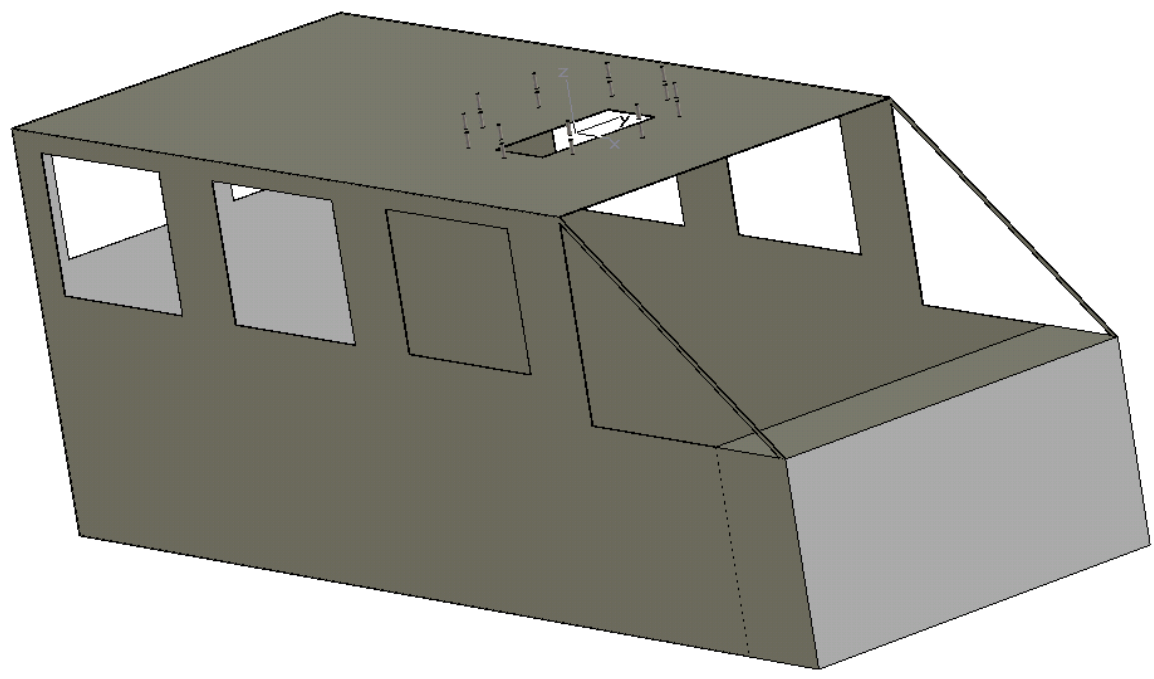

Figure 1. Model of the carrier body (minibus) with a roof hatch.

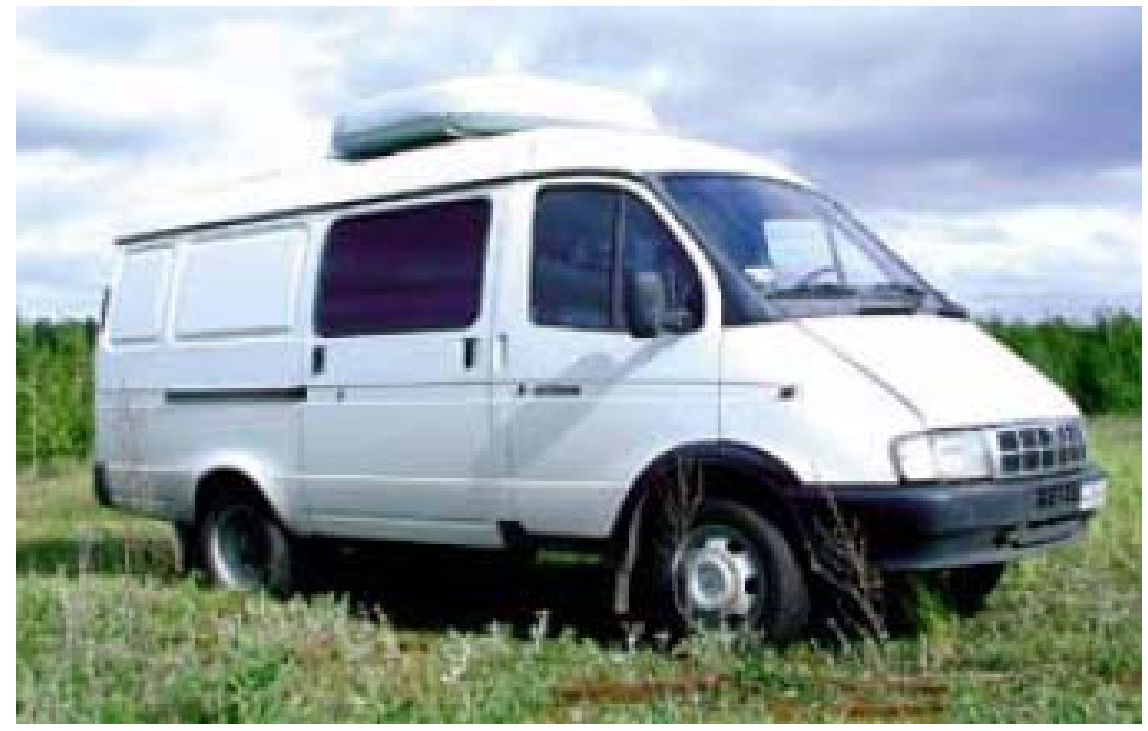

Figure 2. Mobile radio direction finding system of the "Article" family.

Here we consider a method for describing an electromagnetic field measured near the body of mobile carrier of vibrator antenna array, which is based on the decomposition of the observed field into partial components. These partial components are a collection of plane waves, each of which propagates along the lines connecting the vibrators and the array phase center. 
The advantage of such a representation of the field is the reduced sensitivity to random errors in measurement of the field at points corresponding of the array phase centers; the disadvantage is the possibility of reconstructing the field only at $N$ points of the circle $r$, and the radius of this circle can be either larger or smaller than radius of the antenna array $R$.

The results of numerical simulation showed that $E_{z}$-field strength (the $\mathrm{z}$ axis was vertical), measured by the antenna array with radius $R=0.5 \mathrm{~m}$, can be used to approximate the total field (of incident and scattered waves) on circles of radius $r$, which is two or even three times larger than radius of the antenna array $R$. This is illustrated by Figures $3-5$, where radius of the circle for approximation of the field was $r=1 \mathrm{~m}$. The simulation was based on a rigorous solution of diffraction problem of a vertically polarized electromagnetic wave incident (the azimuthal angle between direction of wave incidence and longitudinal axis of the vehicle, in this case, was $\phi_{\text {incidence }}=45^{\circ}$ ) to the analyzed structure "carrier body-antenna array" in the azimuthal plane.

The results of the field approximation on circles with larger radii can be used to reduce the systematic error of DOA estimation; see Figures 6 and 7. The field was restored at a radius of $r=1.5 \mathrm{~m}$ (antenna array radius $\mathrm{R}=0.5 \mathrm{~m}$ ). The dashed lines represent a radiation patterns of the antenna array; solid lines represent similar dependences reconstructed on a circle of three-times-larger radius (bold lines correspond to the phase method of direction finding; thin lines correspond to the amplitude-phase method). The residual error of DOA estimation for the case considered at Figure 6 is $2^{\circ}$ (against the initial error of $16^{\circ}$ ); in case of Figure 7, the residual error is halved (from $6^{\circ}$ to $3^{\circ}$ ).

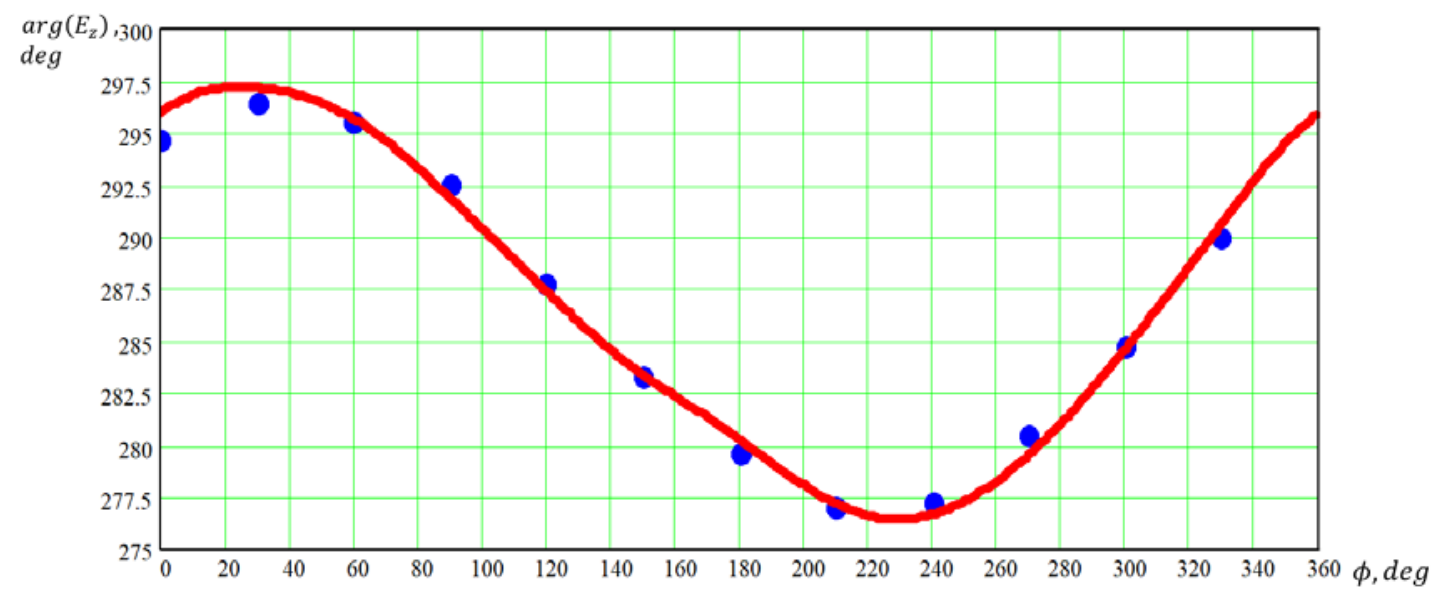

Figure 3. Phase distributions of $E_{z}$-field on a circle with radius $r=1 \mathrm{~m}$ (antenna array radius $R=0.5 \mathrm{~m}$ ) obtained by numerical simulation (circles) and approximation (solid lines) at frequency $f=10 \mathrm{MHz}$.

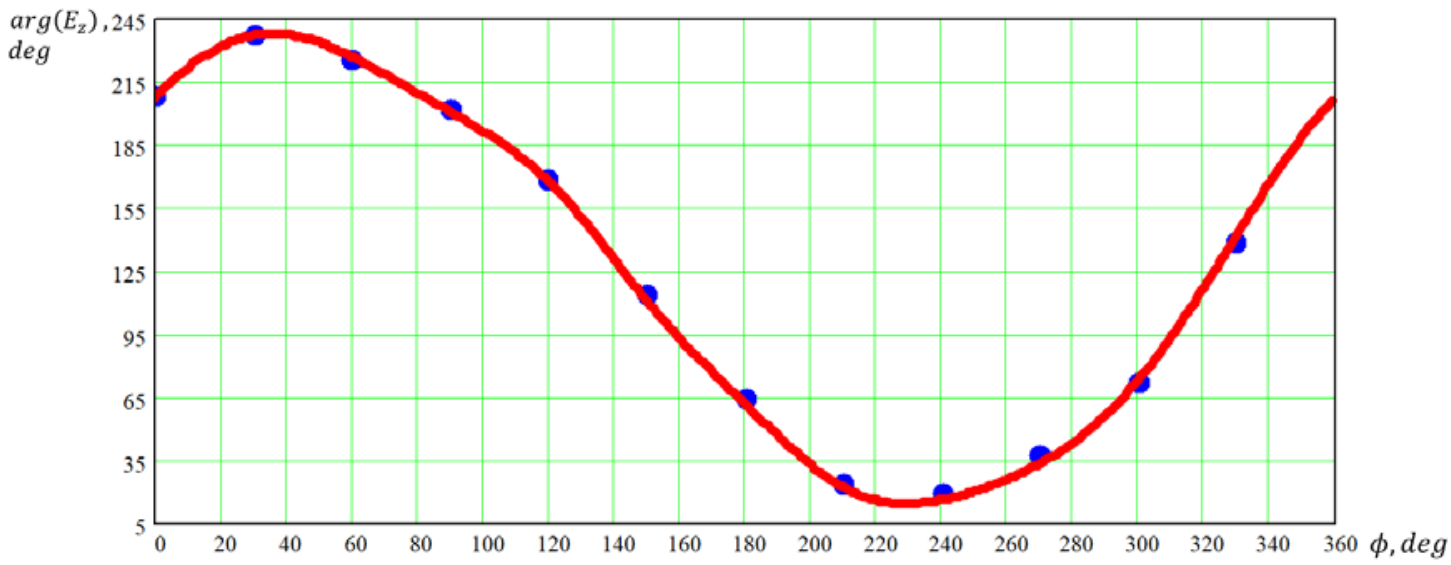

Figure 4. Phase distributions of $E_{z}$-field on a circle with radius $r=1 \mathrm{~m}$ (antenna array radius $R=0.5 \mathrm{~m}$ ) obtained by numerical simulation (circles) and approximation (solid lines) at frequency $f=90 \mathrm{MHz}$. 


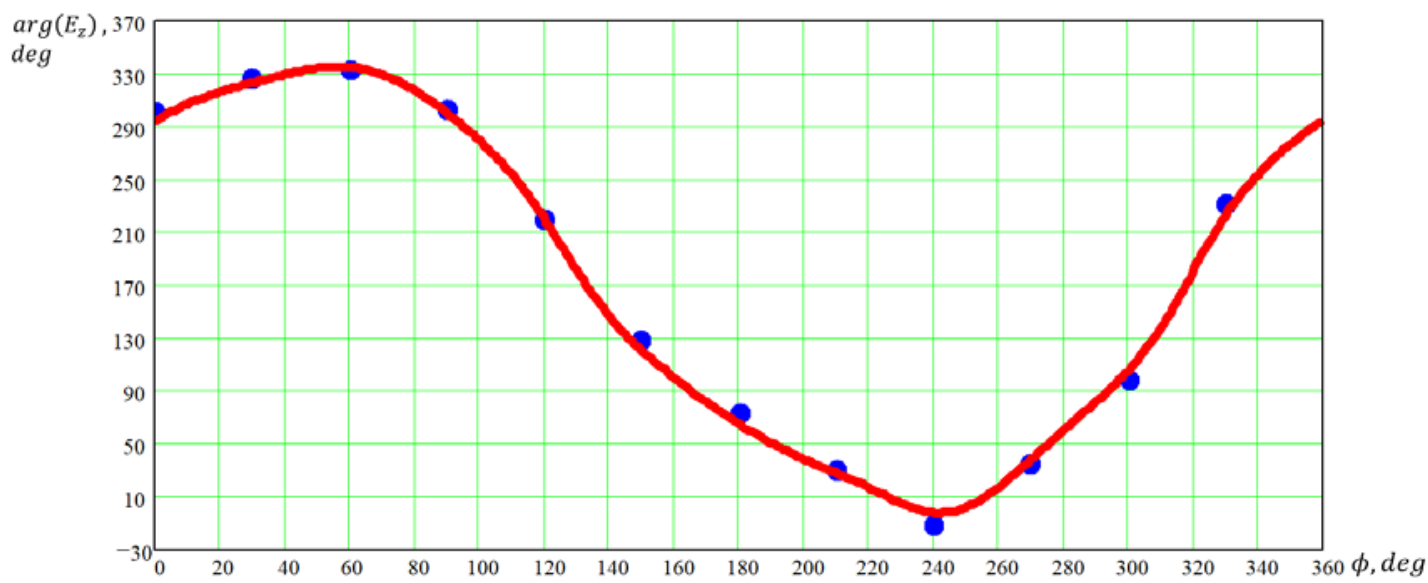

Figure 5. Phase distributions of $E_{z}$-field on a circle with radius $r=1 \mathrm{~m}$ (antenna array radius $R=0.5 \mathrm{~m}$ ) obtained by numerical simulation (circles) and approximation (solid lines) at frequency $f=140 \mathrm{MHz}$.

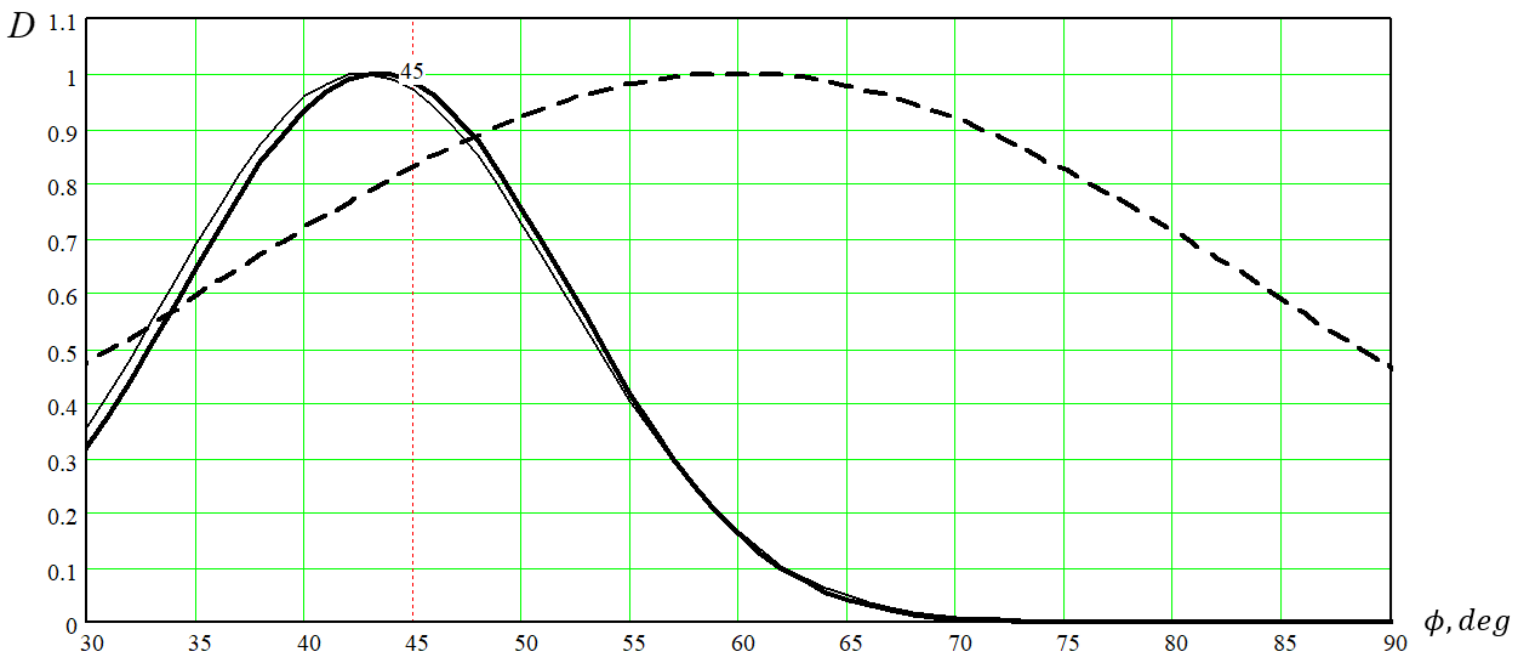

Figure 6. Correction of a Direction of Arrival (DOA) estimation by restoring the phase distribution of the field on a circle: $f=90 \mathrm{MHz}, \phi_{\text {incidence }}=45^{\circ}, \phi_{D O A \text {.rough }}=61^{\circ}, \phi_{D O A \text {.accurate }}=43^{\circ} \mathrm{D}$.

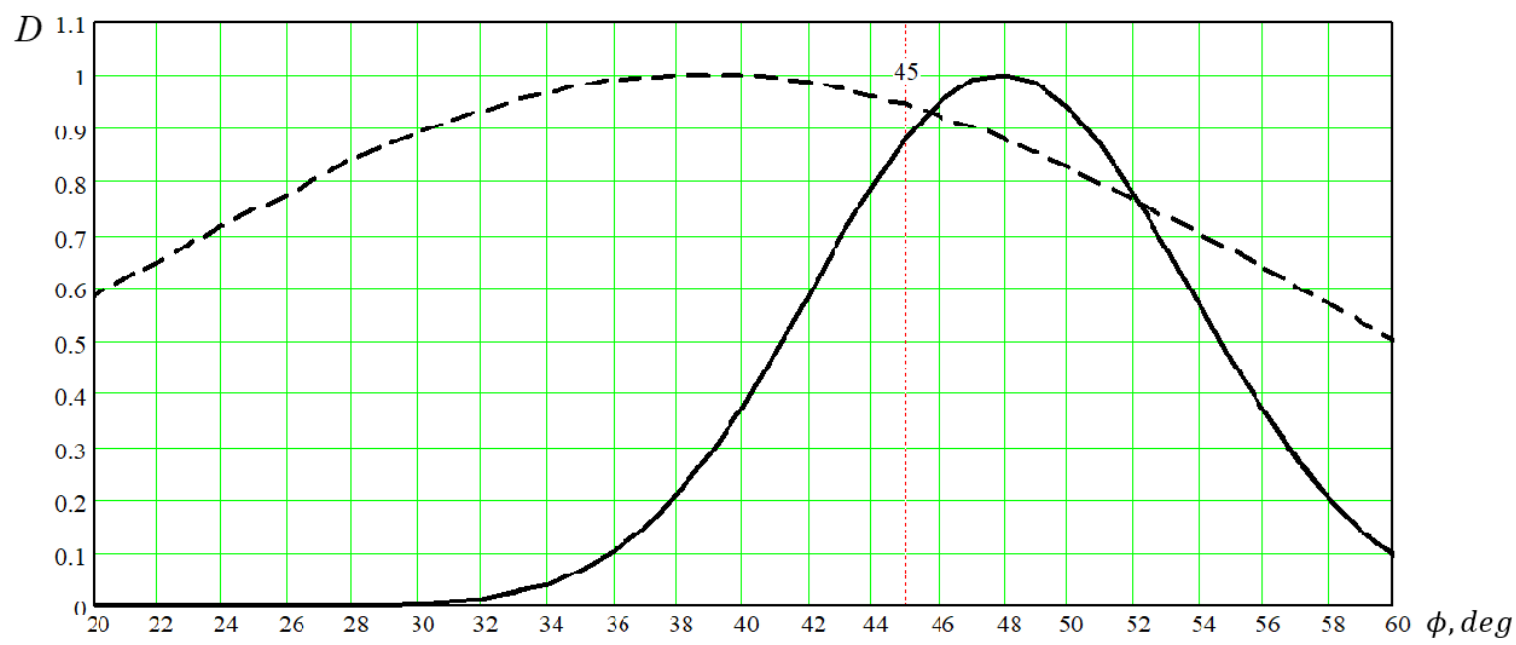

Figure 7. Correction of DOA estimation by restoring a phase distribution of the field on a circle: $f=140 \mathrm{MHz}, \phi_{\text {incidence }}=45^{\circ}, \phi_{D O A}$.rough $=39^{\circ}, \phi_{D O A}$ accurate $=48^{\circ}$. 
The measurement errors of the input signals are random in nature; therefore, their Fourier spectrum is characterized by the concentration of energetically significant harmonic components in the higher frequency region, unlike the Fourier spectrum of the undistorted signal.

The studies showed that the differences in the spectra of the undistorted and distorted signals are maximal at the highest two or three harmonics of their Fourier spectra, while the amplitudes of these harmonics of the undistorted signal are negligible relative to the corresponding spectral components of the distorted signal. This circumstance allows us to use the discrete Fourier transform for filtering higher harmonics, which almost do not carry any useful information about the observed field, and only introduce distortions caused by the influence of noise and measurement errors.

Another effective, although trivial way of compensating the distorting effect of noise and measurement errors of complex amplitudes is to average the measured values over a sufficiently large number of their realizations. Using this selective averaging makes it possible to reduce the maximum value of systematic error of DOA estimation by almost three times (Figure 8).

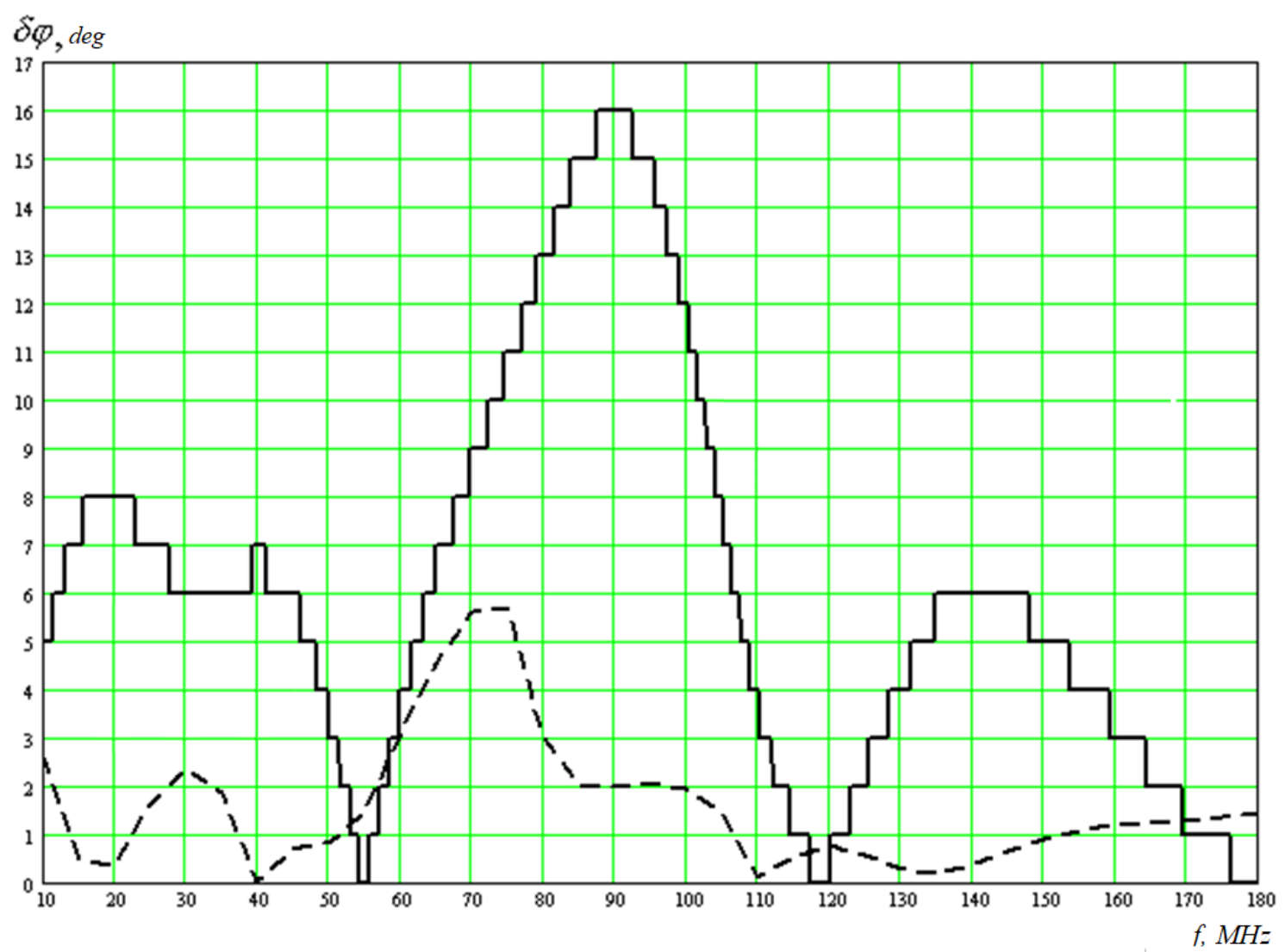

Figure 8. Frequency dependences of DOA errors $\left(\phi_{\text {incidence }}=45^{\circ}\right)$ : the "stepped" line represents the DOA estimated by antenna array of radius $R=0.5 \mathrm{~m}$; the dashed line represents the residual error of DOA estimated by method of auxiliary sources.

\subsection{Method Based on Kirchhoff Integral and Quasisolution}

For the VAA method based on the Kirchhoff integral, as the basic method for representing the field, we chose the method of auxiliary field sources. Taking into account that we were interested in the field values at points located on the same plane as the phase centers of the symmetric electric vibrators of the RAA, we used the scalar Green functions of linear sources as the basis functions for representing the field. We used the quasiresolution procedure to reduce the influence of error of measurements by RAA on accuracy of the field approximation. 
Figures 9 and 10 present the results of an experimental study of a seven element antenna array with a radius of $500 \mathrm{~mm}$ located on the roof of the GAZelle minibus, with true direction to radio sources equal to 30 degrees (Figure 9) and 60 degrees (Figure 10). The angles are counted counterclockwise; zero angle is directed towards the vehicle cabin.

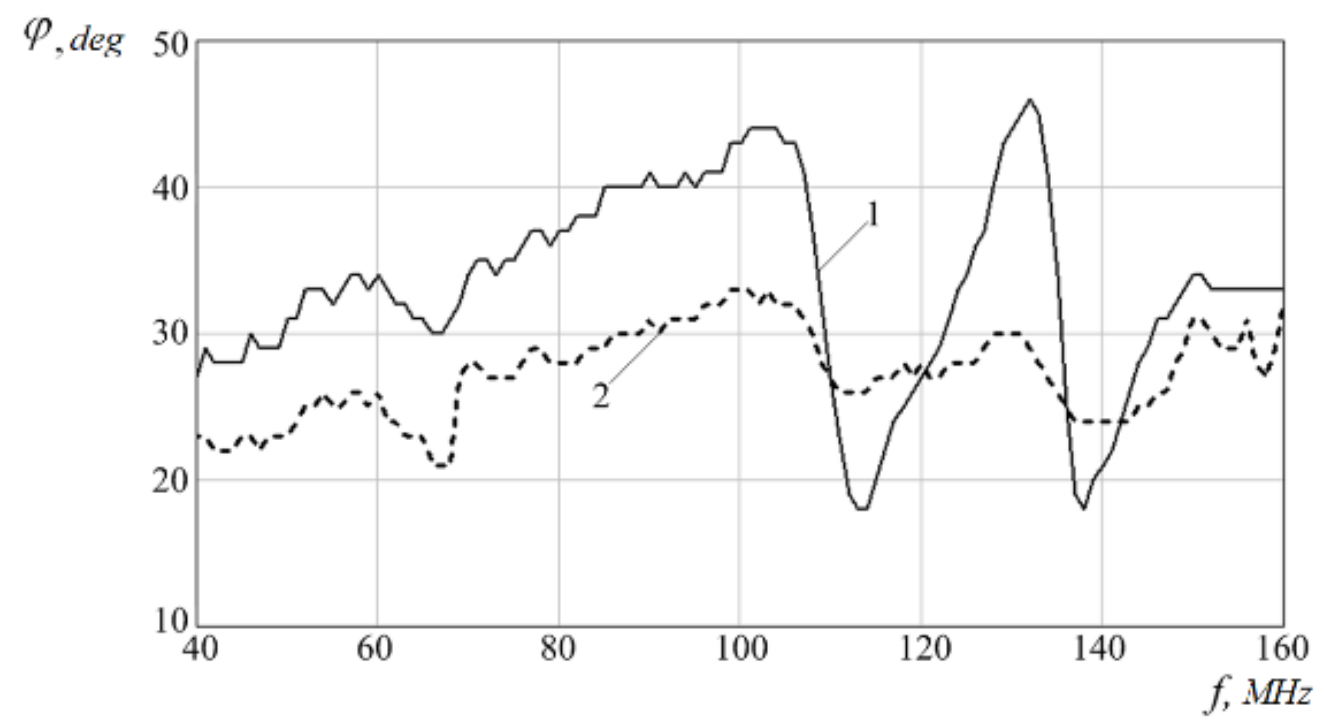

Figure 9. Experimental frequency dependences of the DOA estimates with true angle of arrival $30^{\circ}$. Curve 1-correlation-interferometric method; Curve 2-the VAA method.

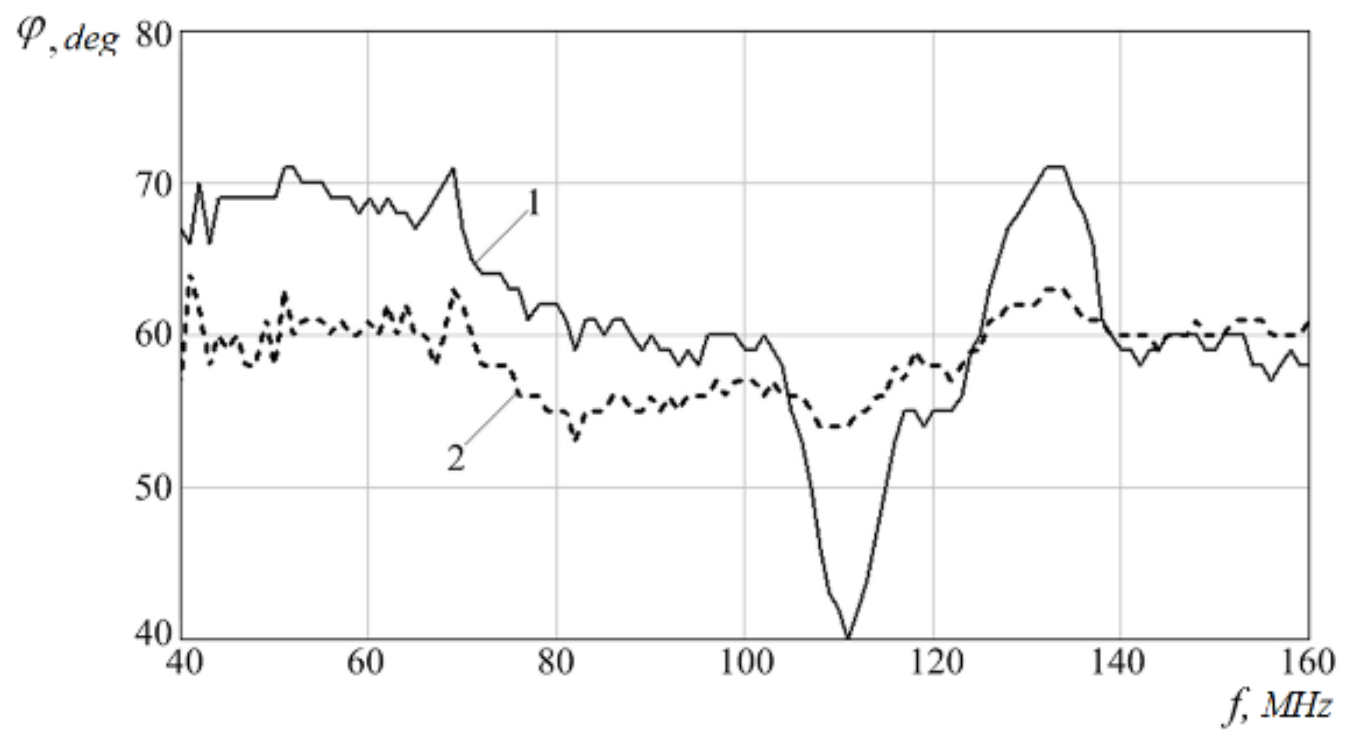

Figure 10. Experimental frequency dependences of the DOA estimates with true angle of arrival $60^{\circ}$. Curve 1-correlation-interferometric method; Curve 2-the VAA method.

The bearings were calculated by two methods: the interference-correlation method (Curve 1) and the VAA method in conjunction with the quasisolution procedure (Curve 2). These dependences show that use of VAA can not only eliminate gross errors in DOA estimation, but also generally reduce the DOA estimation error in the studied frequency range. Maximum error of DOA estimation is decreased up to four times and the standard error of DOA estimation decreases by up to two times. 


\subsection{Method Based on Laurent Series}

Numerical experiments have shown that Laurent series expansion of $E_{z}$-field inside a circle with a radius equal to twice the radius of the antenna array $(r=2 R)$ can increase the instrumental accuracy of a mobile-based radio direction finder up to 1.4-1.5 times. An effective way of forming a VAA is a numerical estimation carried out using the Loran series (7); several derivatives of the function $U(z)$ in radial directions $\frac{\partial U(\phi, r)}{\partial r}, \frac{\partial^{2} U(\phi, r)}{\partial r^{2}}$, etc.; and estimation of values of the function $U(\phi, R+L)$, where $L$ can exceed the antenna array radius $R$, with the use of a segment of the Taylor series.

Figures 11-14 present the frequency dependences of DOA estimation and its absolute errors, corresponding to the traditional use of RAA with radius $R=0.54 \mathrm{~m}$ and the number of elements $N=7$ (solid bold lines), and VAA with radius $r=1.24 \mathrm{~m}$ and number of "elements" (approximated point samples of the field) $N=12$ (lines with circles). In the numerical simulation, the Taylor series (8) was truncated to its first three terms.

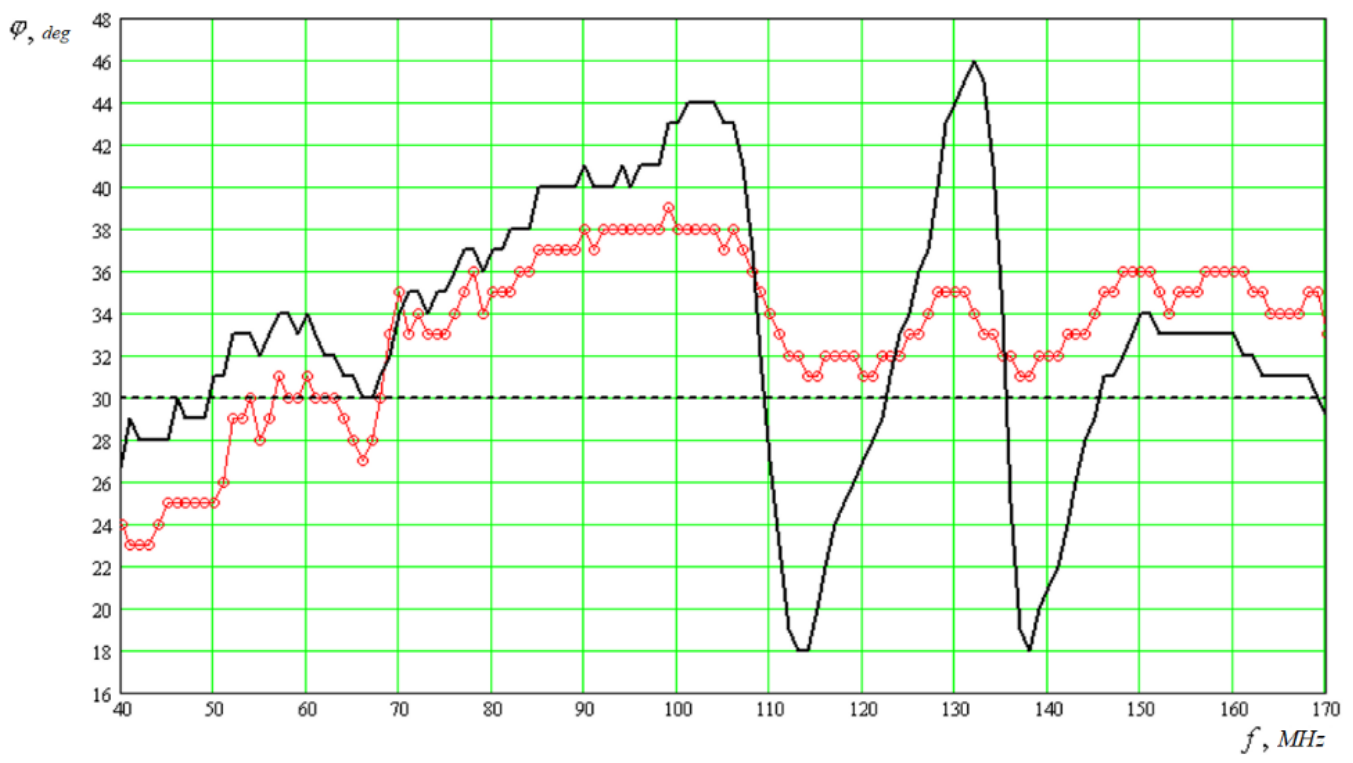

Figure 11. Frequency dependences of DOA estimation for the true azimuth of radio source $\phi_{\text {source }}=30^{\circ}$.

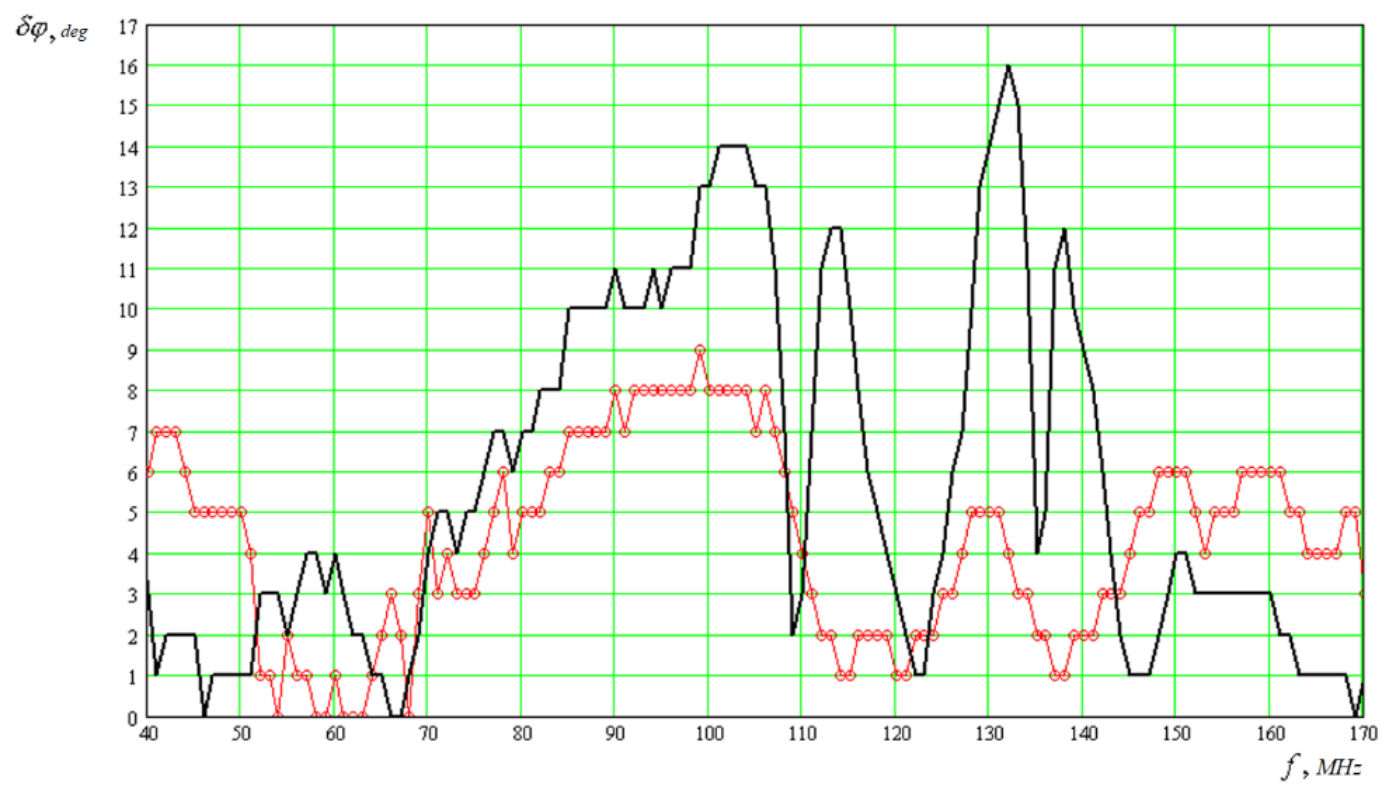

Figure 12. Frequency dependences of absolute error of DOA estimation for the true azimuth of radio source $\phi_{\text {source }}=30^{\circ}$. 


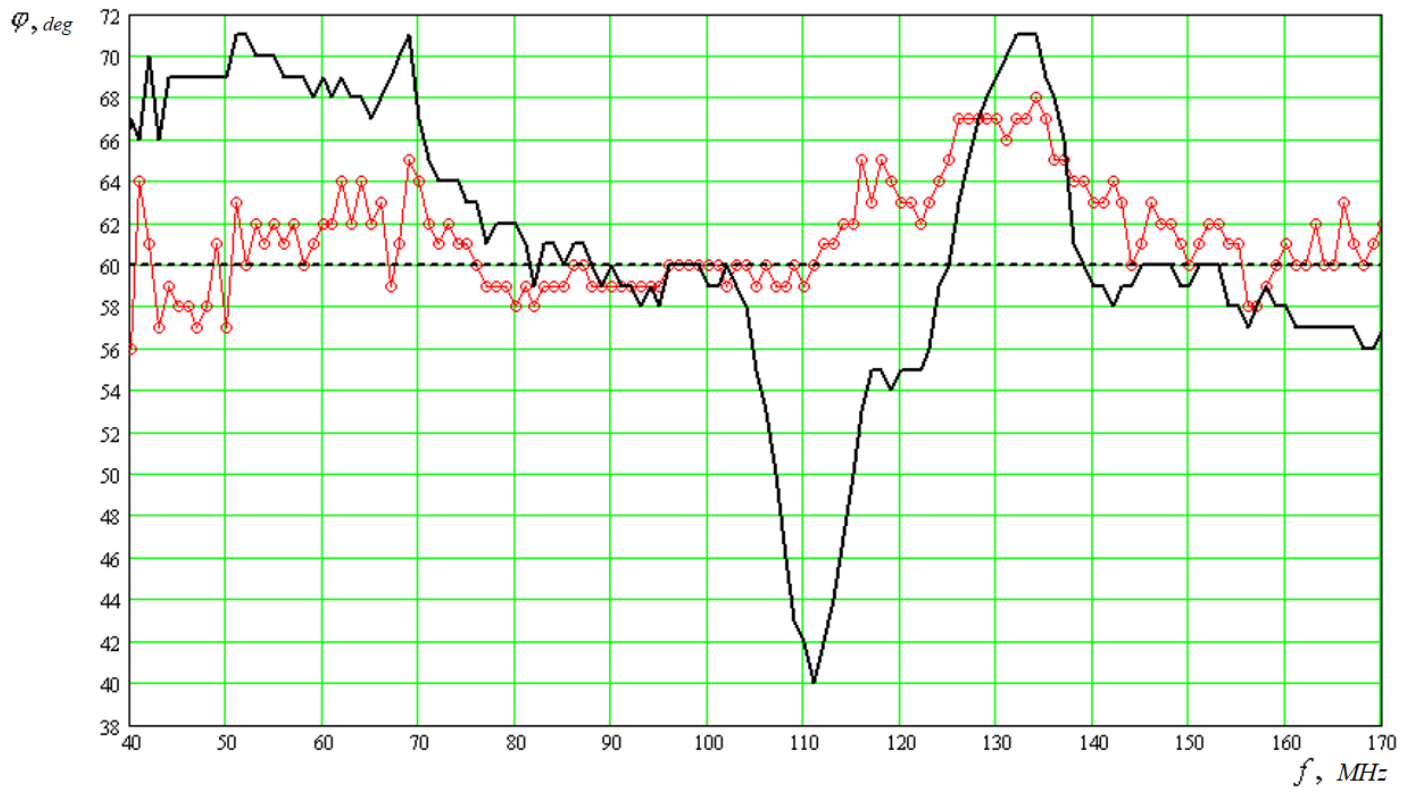

Figure 13. Frequency dependences of DOA estimation for the true azimuth of radio source $\phi_{\text {source }}=60^{\circ}$.

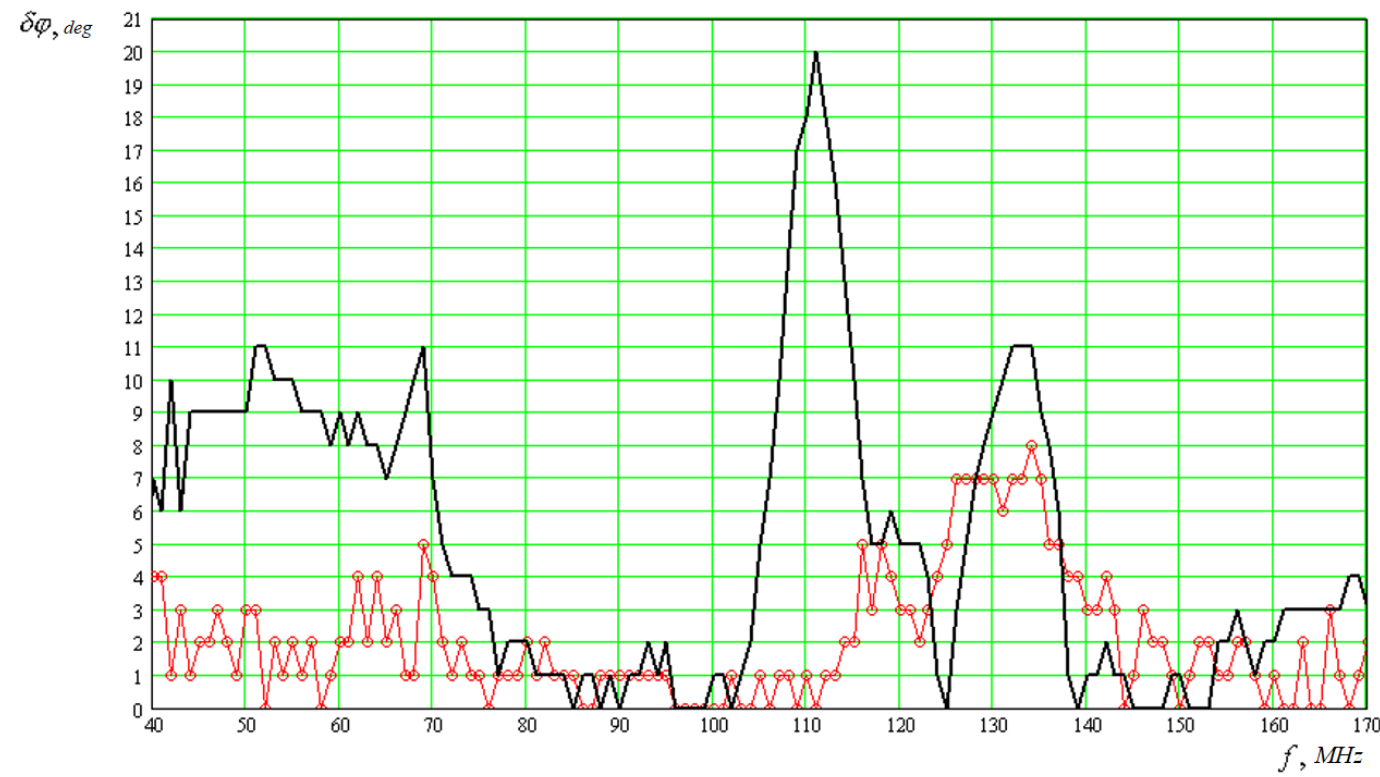

Figure 14. Frequency dependences of absolute error of DOA estimation for the true azimuth of radio source $\phi_{\text {source }}=60^{\circ}$.

The figures show that VAA is most effective in a frequency band corresponding to the most intense resonances of the antenna array carrier body-from 80 to $145 \mathrm{MHz}$. When true azimuth of the radio source $\phi_{\text {source }}=30^{\circ}$, the maximum error of DOA estimation, in the frequency band from 40 to $170 \mathrm{MHz}$, decreases by 1.778 times (from $16^{\circ}$ to $9^{\circ}$ ) due to VAA; the standard error of DOA estimation decreases by 1.47 times (from $7.183^{\circ}$ to $4.887^{\circ}$ ). Additionally, when true azimuth of the radio source $\phi_{\text {source }}=60^{\circ}$, the maximum error of DOA estimation decreases by 2.5 times (from $20^{\circ}$ to $8^{\circ}$ ) due to VAA; the standard error of DOA estimation decreases by 2.38 times (from $6.801^{\circ}$ to $2.858^{\circ}$ ).

\subsection{Method Based on Auxiliary Field Sources Applied to a Stationary Antennas}

Figure 15 shows a model of the ARK-MK4 stationary antenna array (mounted on a telescopic mast with maximum height of $10 \mathrm{~m}$ ), which is part of the ARK-MK1M radio monitoring complex, 
as shown in Figure 16. A high-frequency switching unit and two-channel receivers are located in the center of the antenna array. Stable functioning of this unit in various climatic conditions was ensured by installing an automatic temperature stabilization. A radio receiver unit was placed in close proximity to the array vibrators to minimize power losses in feeder lines, and increase the sensitivity and noise immunity of the radio monitoring complex. However, a rather large additional scatterer (radio receiver unit dimensions are approximately $500 \times 500 \times 180 \mathrm{~mm}^{3}$ ) can adversely affect the accuracy and unambiguity of DOA estimation.

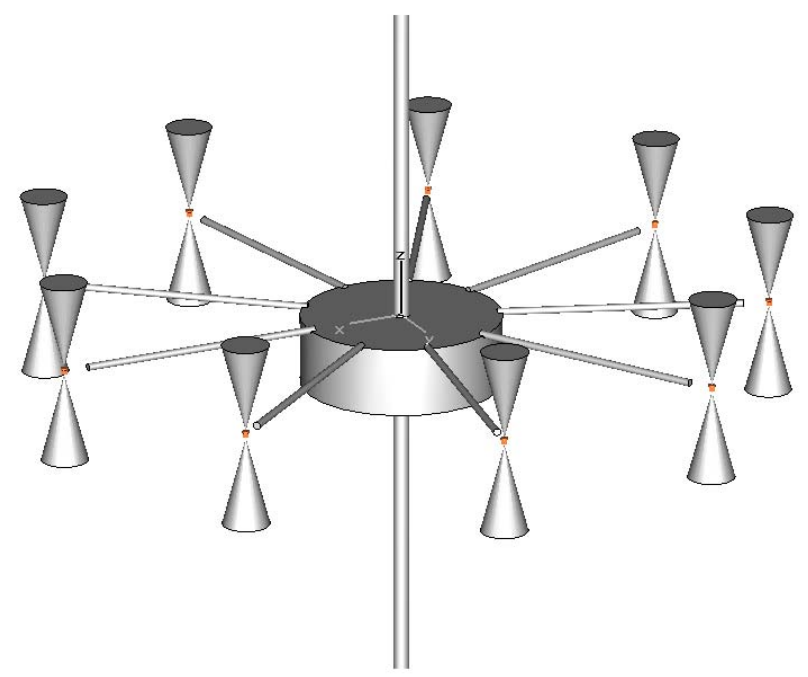

Figure 15. Model of a stationary antenna array: array radius $R_{R A A}=1 \mathrm{~m}$; number of elements $N=9$; vibrator length $2 \cdot L=0.5 \mathrm{~m}$; vibrator base diameter $D_{b}=0.128 \mathrm{~m}$; vibrator load resistance $R_{\text {load }}=1000$ ohms.

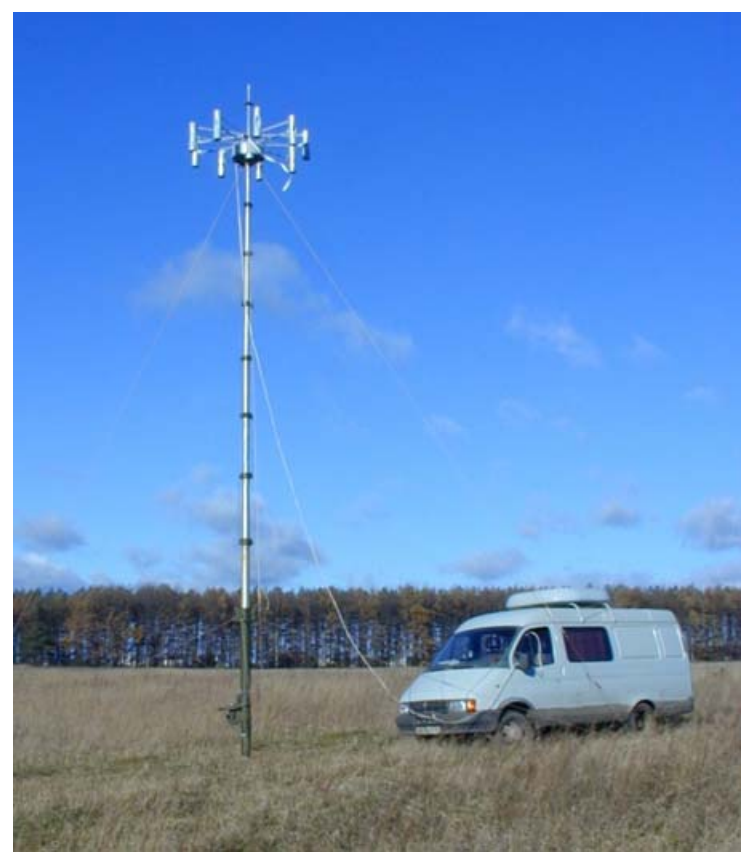

Figure 16. The ARK-MK1M radio monitoring complex with an additional antenna and radio receiving system ARK-MK4 on a telescopic mast.

The receiving antenna system (Figure 15) has several self-resonances because of its complex geometry and ultra-wide frequency band (from $25 \mathrm{MHz}$ to $1000 \mathrm{MHz}$ ). Electromagnetic waves are scattered on antenna elements, the antenna mast, the switching unit and receiver, the horizontal bars, 
the guy-wires, and other nodes of the receiving antenna system. That can lead to significant distortion of the observed field, and therefore to gross errors of DOA estimation: for example, "inversion" of DOA estimation caused by the diffraction back lobe of a radiation pattern which is exceeding the level of main lobe. In this case, the proposed method for approximation of an observed electromagnetic field as plane waves propagating along the lines connecting the vibrators and array phase center can eliminate gross errors of DOA estimation, which is illustrated in Figures 17 and 18.

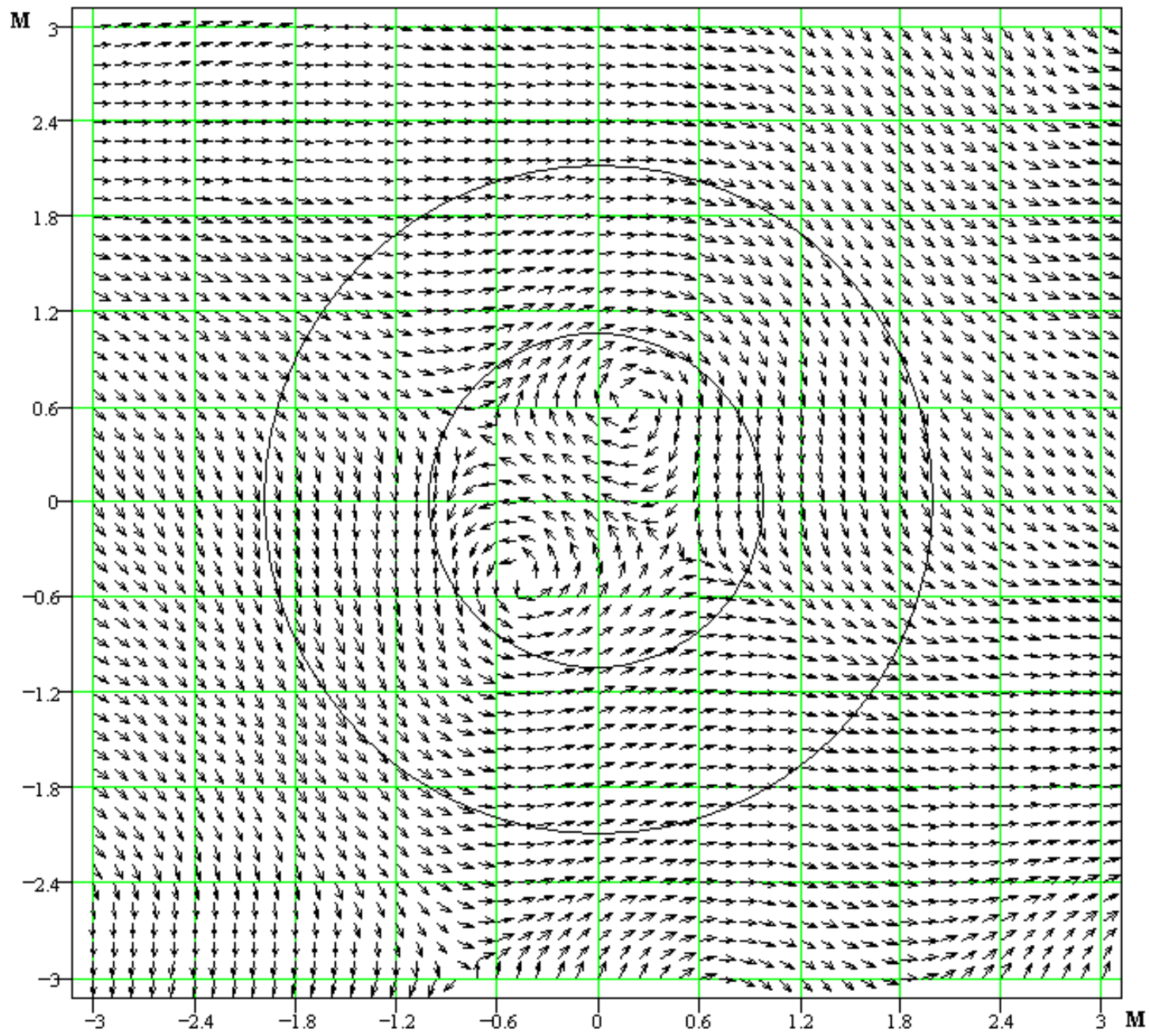

Figure 17. The spatial distribution of the "differential" DOA estimated by an antenna array of 9 elements mounted on a mast; $\phi_{\text {incidence }}=330^{\circ}$ and $f=62 \mathrm{MHz}$ (at this frequency, due to distortion of measured field structure, there is an "inversion" of DOA estimation-the back lobe of radiation pattern becomes higher than the main lobe); the inner circle corresponds to Real Antenna Array (RAA) $\left(R_{R A A}=1 \mathrm{~m}\right)$; radius of the outer circle is $2 \mathrm{~m}$ (radius of Virtual Antenna Array (VAA)); in the center of the figure, we can see a turn of the "differential" DOA estimation by $180^{\circ}$. 


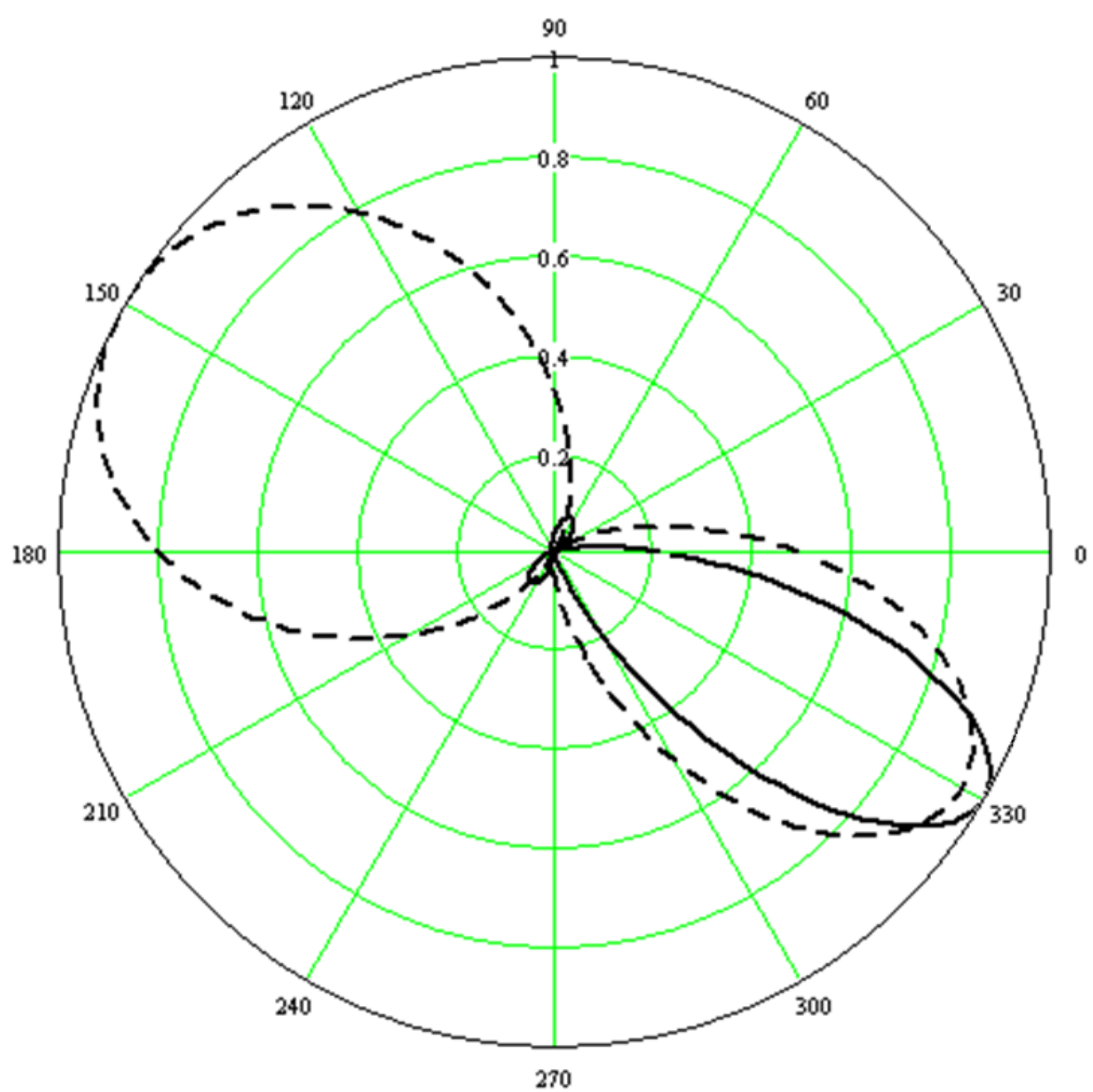

Figure 18. The radiation patterns of RAA (dashed line) and VAA (solid line) at a frequency of $f=62 \mathrm{MHz}$.

Spatial distribution of the "differential" DOA estimation (Figure 17) shows that inside antenna array the field has a vortex pattern, which is due to the scattering on the switching unit and receiver, and therefore, lines of the "differential" DOA estimation are turned by $180^{\circ}$ in the center of the antenna array. As a result of the back lobe increasing to a level higher than the main lobe, a DOA error of $180^{\circ}$ occurs; see Figure 18 (dashed line). The field reconstructed on circle with radius twice as large as RAA can be used to calculate the DOA curve and avoid gross error of DOA estimation (Figure 18, solid line). Additionally, the back lobe of radiation pattern is completely absent-there are only two side lobes symmetrically located on both sides of main lobe; in addition, the resolution of antenna array is increased due to double radius of VAA.

The stability of the VAA method's results was studied by taking into account errors in measurement of amplitudes and phases which can be caused by various factors-systematic errors in measurement of signal parameters, differences in feeder lines, and the effects of noise and interference. It was assumed that the mobile-based receiving antenna system (mounted on the roof of carrier body, shown in Figure 2) operates at high input signal-to-noise ratios (more than 25-30 dB), that the error in measuring amplitudes of received signals is not more than $5 \%$, and the standard deviation of the phase measurement is $2.5^{\circ}$. DOA estimations were averaged over 1000 results of their independent statistical tests. 


\subsection{Increase of Resolution of Direction Finders by VAA Method}

One of the applications of VAA is to increase the resolution of direction finders. In this article, a theoretical case was considered: scatterers were not taken into account; an electromagnetic wave was propagating in a vacuum. The observed field on the $(x 0 y)$ plane, if there are no scatterers, is a superposition of the fields of sources $\dot{U}_{\Sigma}(x, y)=\dot{U}_{1}(x, y)+\dot{U}_{2}(x, y)$.

Such a simplification is acceptable for studies of the resolution of two radio sources in a first approximation. The rationale for this thesis was from the results obtained through studies of the effectiveness of VAA in the case of changing the geometry of the carrier body of a mobile direction finder.

The following model parameters were considered: the radius of RAA, consisting of nine elements, was $0.5 \mathrm{~m}$; the amplitudes of the sources of electromagnetic waves were $A_{1}=1$ and $A_{2}=2$; the initial phases of the sources were chosen to be equal to $\varphi_{1}=0, \varphi_{2}=\pi / 2$; angular distance $\left|\alpha_{1}-\alpha_{2}\right|$ between azimuths of the sources of electromagnetic waves was chosen to be comparable to the width of the main lobe or less, so these sources were not resolvable by the main lobe scanning in the azimuthal coordinate. The formation of VAA was carried out using a method based on the superposition of the fields of point auxiliary sources.

Figures 19 and 20 present the results of resolving two radio sources with true azimuthal coordinates $\alpha_{1}=80^{\circ}$ and $\alpha_{2}=180^{\circ}$ (not resolvable by RAA) using a VAA with a radius 2.5 times greater than RAA. VAA consisted of 72 extrapolated field samples for a source frequency of $100 \mathrm{MHz}$.

In Figures 19 and 20, the dashed lines show the normalized directivity patterns of RAA; solid lines-VAA.

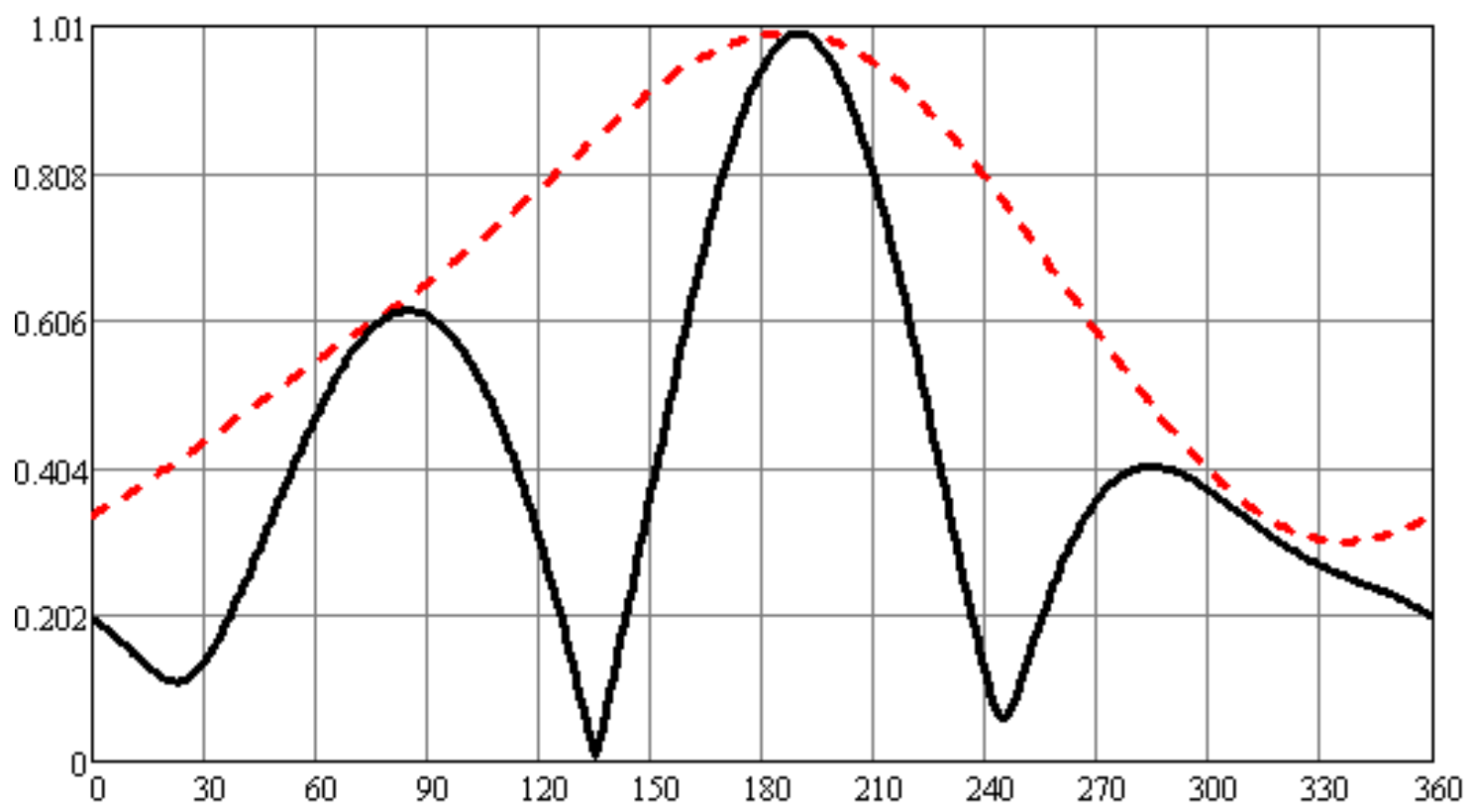

Figure 19. Azimuths of the sources $-\alpha_{1}=80^{\circ}$ and $\alpha_{2}=180^{\circ}$; VAA radius is $1.25 \mathrm{~m} ; \mathrm{N}=72$. 


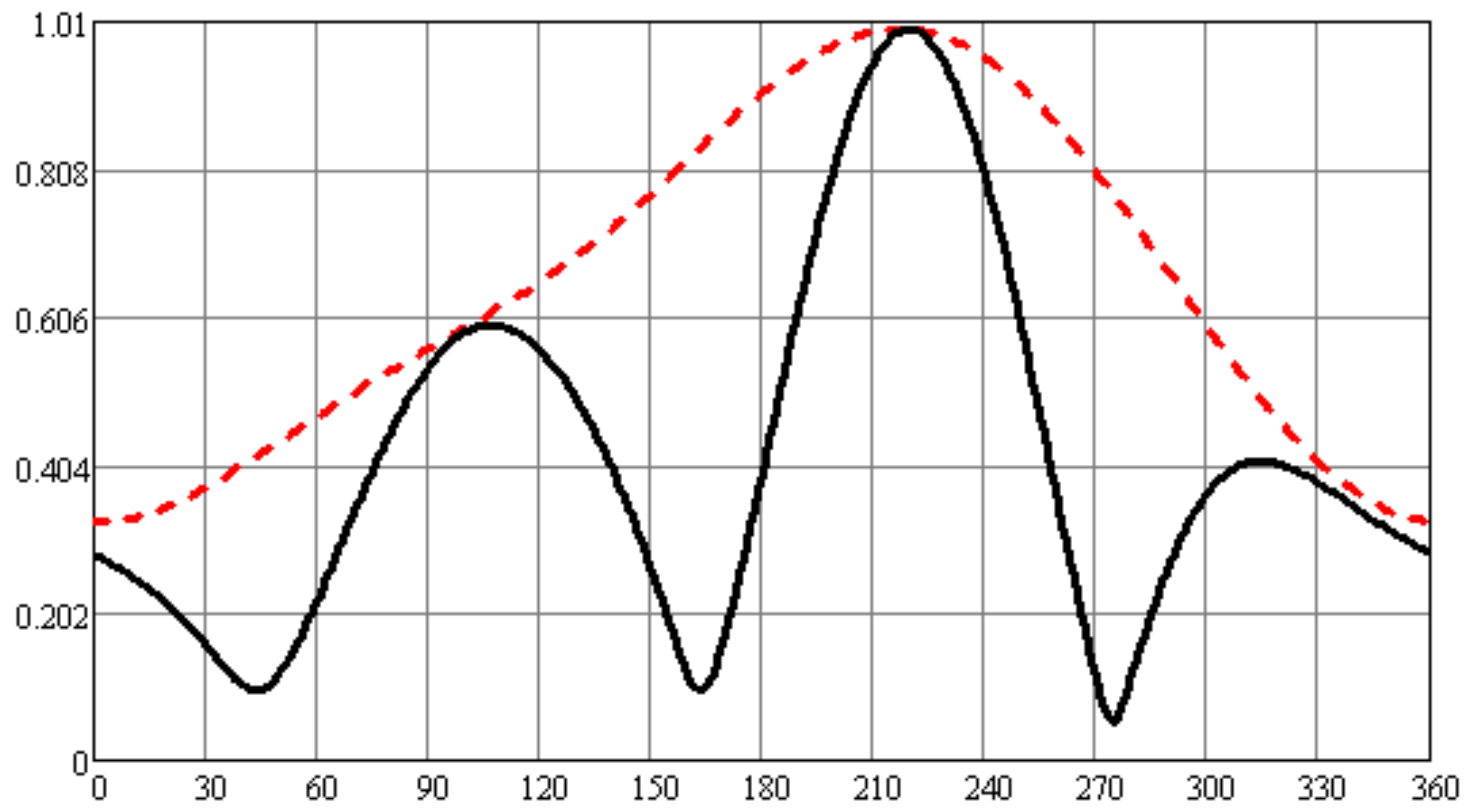

Figure 20. Azimuths of the sources $-\alpha_{1}=100^{\circ}$ and $\alpha_{2}=220^{\circ}$; VAA radius is $1.25 \mathrm{~m} ; \mathrm{N}=72$.

Figures 19 and 20 show that the formation of VAA with a radius 2.5 times larger than RAA allows one to determine the true number of sources and estimate their angular coordinates, while in the radiation pattern of the RAA there is only one maximum.

Additionally, we study the possibility of using the method of the Laurent series to approximate the spatial distribution of electromagnetic field in the ring, inside which there is a circle of the RAA elements.

We only note the main stages of this approach to extrapolating the spatial distribution of the electromagnetic field, and as a result the generated spatial samples, are interpreted by us as "elements" of some VAA. The field measured by RAA was interpolated on the antenna elements circle by the Kotelnikov series.

Next, the interpolated field samples were used to find the coefficients of the Laurent series describing some analytic function of the complex variable $z=x+i y$ in the ring. This function was considered to be a quasistatic approximation of the spatial distribution of the electromagnetic field (since an antenna array diameter of $1 \mathrm{~m}$ is less than the wavelength in free space three times at a frequency of $100 \mathrm{MHz}$ ).

The next step is to find the derivatives of the field in radial directions connecting the center of the array with the coordinates of the extrapolated field samples, and extrapolating the field using a segment of the Taylor series. The obtained values of the spatial samples of the field formed on a circle with a radius of $1.9 \mathrm{~m}$ (radius of RAA consisting of nine elements equal to $0.5 \mathrm{~m}$ ) were interpreted as elements of VAA.

Figure 21 shows the dependences of normalized radiation patterns of RAA and VAA. The dashed lines show the normalized patterns for RAA, the solid lines for VAA formed without using the field approximation on the RAA circle using the Kotelnikov series, and the dot-and-dash lines for VAA formed using such a field approximation. 


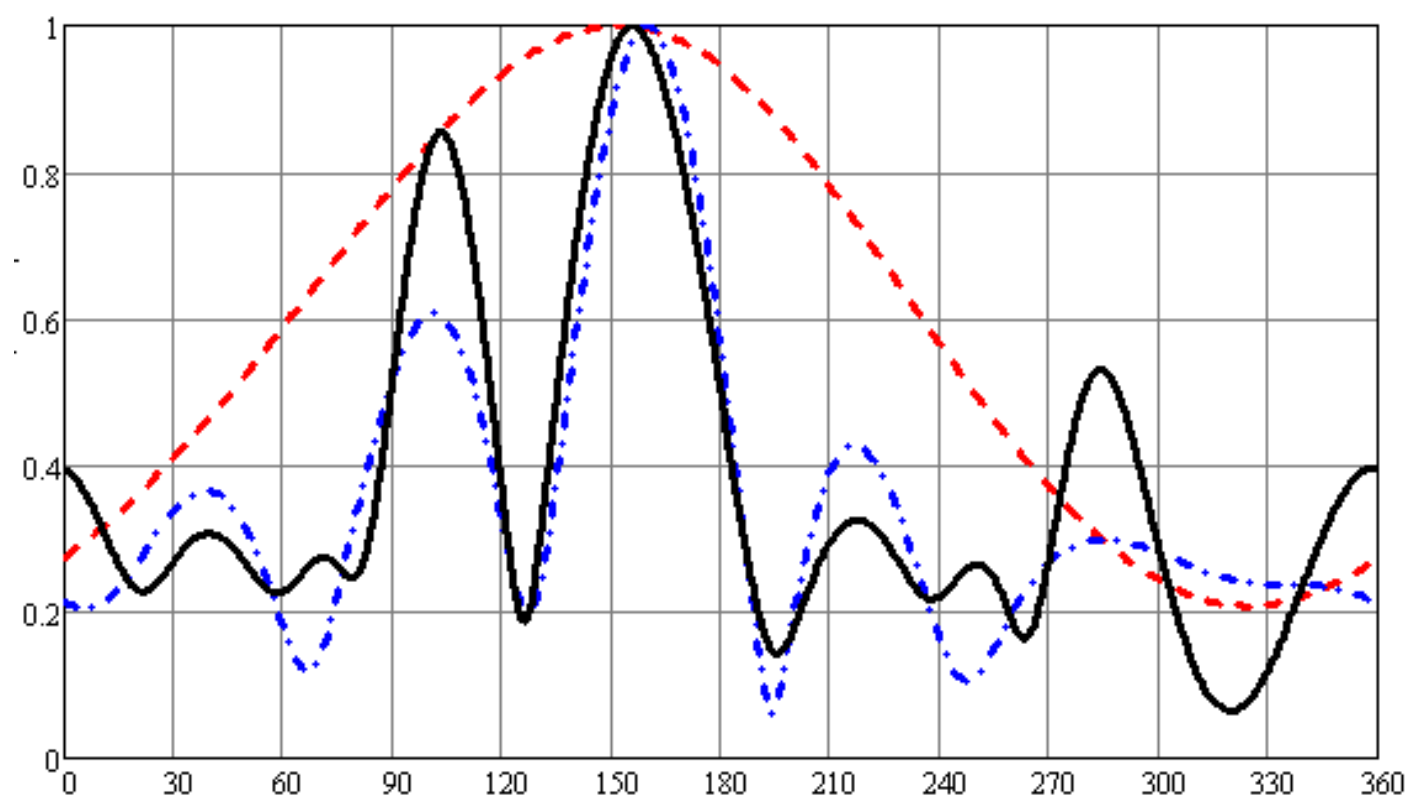

Figure 21. Azimuths of the sources $-\alpha_{1}=100^{\circ}$ and $\alpha_{2}=160^{\circ}$; VAA radius is $1.9 \mathrm{~m} ; \mathrm{N}=72$.

Figure 21 shows that by use of the VAA, radio sources can be resolved, while in case of the RAA, the sources are not resolvable, because the angular distance between the sources is $60^{\circ}$ which is significantly less than the width of the main lobe: $\Delta \alpha_{0.5 P_{0}}=51^{\circ} \frac{\lambda_{0}}{d}=51^{\circ} \frac{3}{2 \cdot 0.5}=153^{\circ}$.

Thus, the obtained results of numerical modeling allow us to conclude that the VAA method has potential for increasing the resolving powers of radio direction finders.

\subsection{Formation of VAA near a Mobile Phone}

It is promising to measure the field with a compact antenna system of a mobile phone, and then restore the field values in the area of space located outside the handset, where the head and hand of the user do not screen and scatter the received waves. The approximated complex field amplitudes can be interpreted as elements of a VAA and can be used for adaptive processing of received information: refinement of the angular coordinates of radio sources, interference suppression, increasing the signal-to-noise ratio at the receiver input, and increasing the resolution abilities in angular coordinates. A key feature of the method is that it does not use any information about the scatterer (user's body), and another is the high efficiency of its implementation in a portable computer system for real-time processing.

It should also be noted that use of the VAA method would significantly increase the efficiency of superresolution methods for radio sources and adaptive signal processing methods in multichannel systems (in particular, the MIMO method) due to the possibility for an increase of virtual channels which are functionally equivalent to additional real channels, and an increase in the electrical dimensions of the antenna system.

The electromagnetic field of the incident wave was measured at 18 points located equidistant around a circle with a radius of $R_{R A A}=15 \mathrm{~mm}$ near the handset. These field readings were taken as field samples measured by the handset antenna. In addition, complex amplitudes of the E-component were measured on circles with radiuses of $R_{1}=22.5 \mathrm{~mm}, R_{2}=30 \mathrm{~mm}$, and $R_{3}=45 \mathrm{~mm}$ at 18 equidistantly spaced points. The human head model was presented in the form of a two-layer structure [36]: the outer layer imitated human skin $(\varepsilon=5, \sigma=0.0125 \mathrm{~S} / \mathrm{m}, \operatorname{tg} \delta=0.05)$, and the inner layer was presented as liquid ( $\varepsilon=42, \sigma=0.99 \mathrm{~S} / \mathrm{m}$,). The model of the phone was simplistically represented by a parallelepiped made of ideal metal with dimensions of $100 \times 40 \times 18 \mathrm{~mm}$, Figure 22 . 


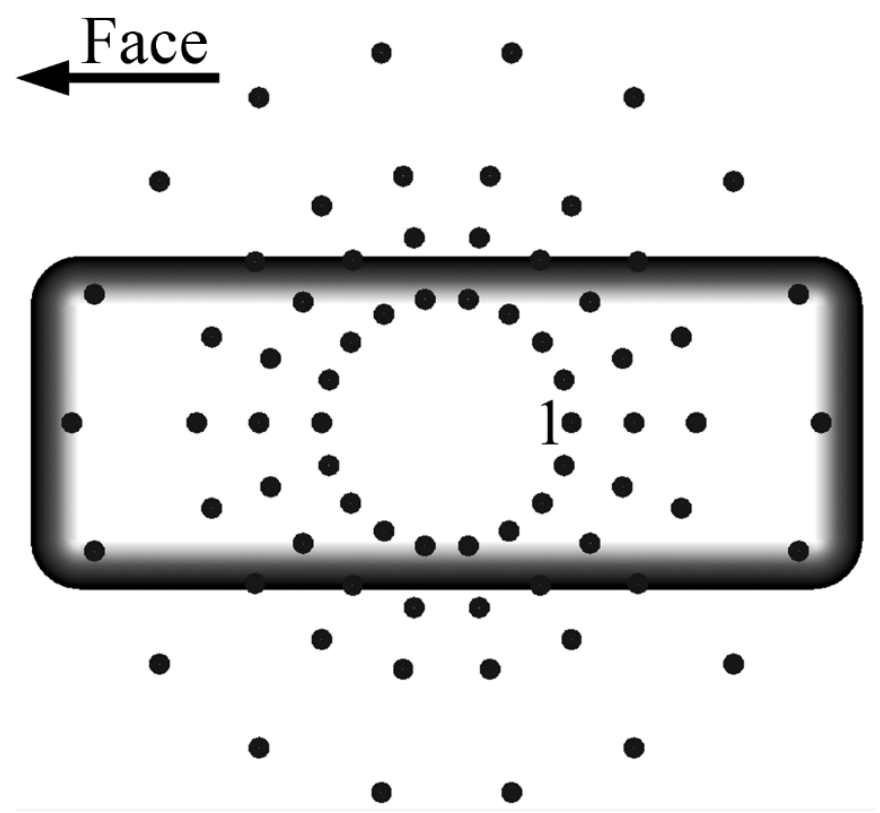

Figure 22. Location of spatial readings in which EMF (electromagnetic field) was recorded relative to the phone case.

Using the values of complex amplitudes measured on the radius $R_{1}$, the phase values were predicted at the corresponding points, on the circles $R_{2}, R_{3}$. Figures 23 and 24 show graphical dependencies of the predicted phases. The circles indicate the phase values at each point measured experimentally; the rhombs-the phase values obtained by approximation.

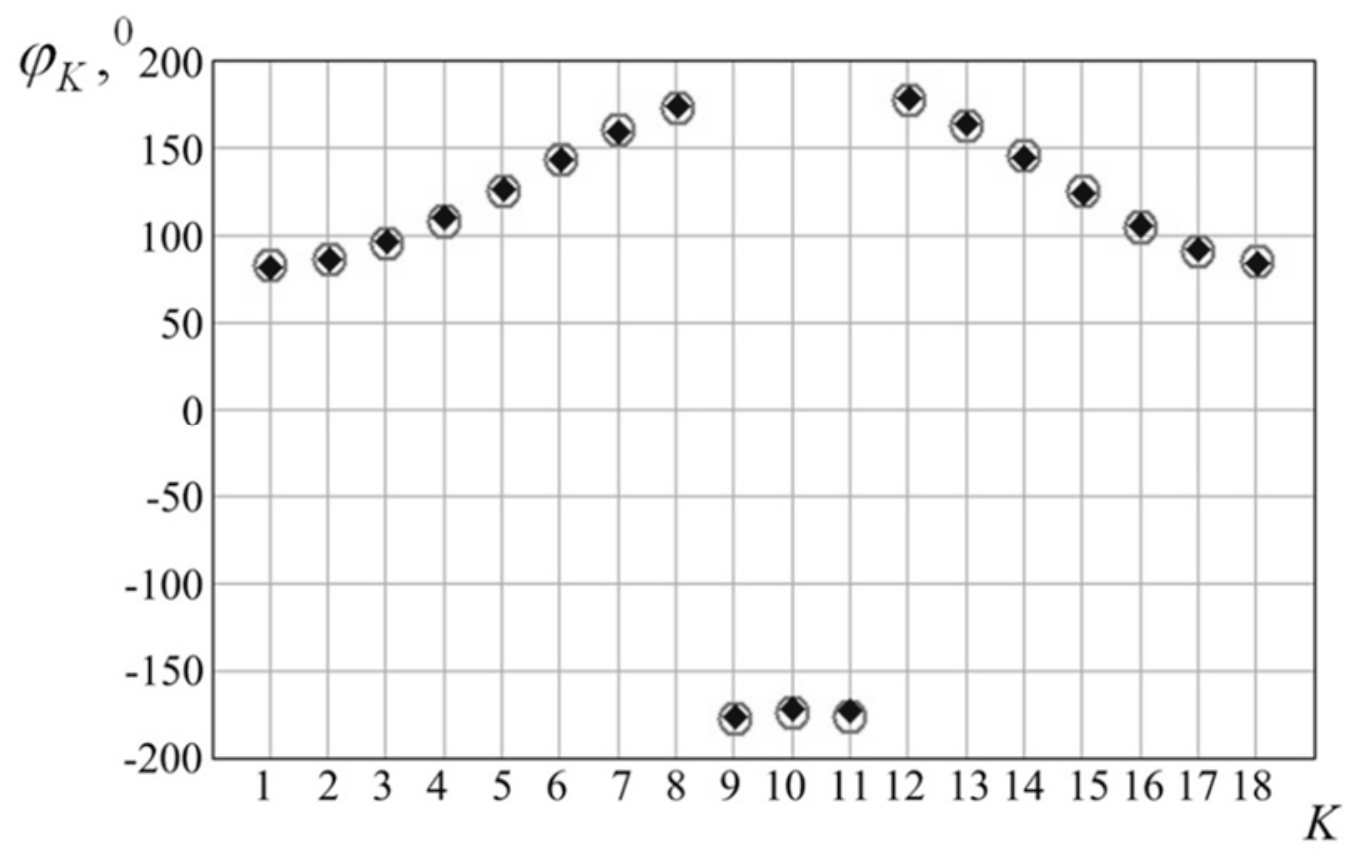

Figure 23. The phase values at points on the radius $R_{1}=22.5 \mathrm{~mm}$ at $f=1800 \mathrm{MHz}$. 


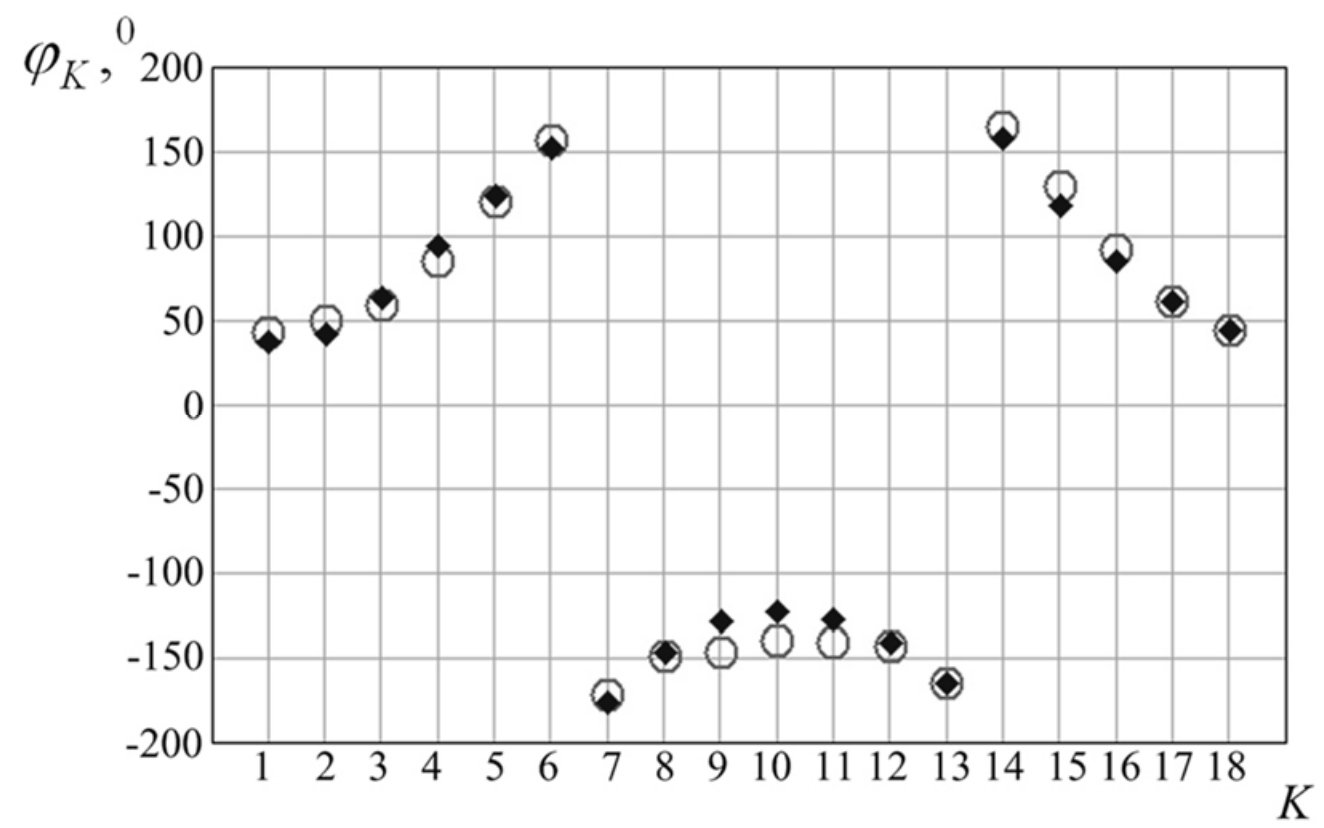

Figure 24. The phase values at points on the radius $R_{1}=45 \mathrm{~mm}$ at $f=1800 \mathrm{MHz}$.

As can be seen from the presented data, the use of the VAA method allows us to quite successfully approximate the phase structure of the electromagnetic field at distances up to three times the radius of the RAA which were used to receive electromagnetic waves.

\subsection{Formation of VAA Consisting of Magnetic Vibrators}

The model of the physical part of the proposed vector antenna element is shown in Figure 25. It consists of 12 symmetric electric vibrators placed along the edges of an electrically small cube. A series connection of a resistance of $50 \mathrm{ohms}$ was used; a capacitance of $1.5 \mathrm{pF}$ was used as a load, which characterizes the input resistance of the antenna buffer.

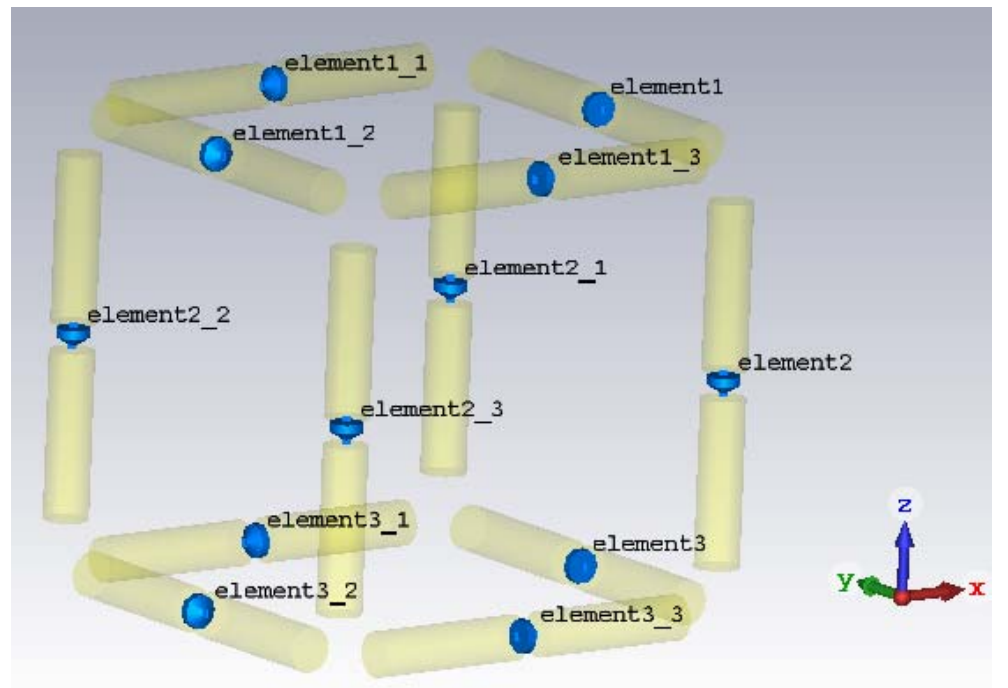

Figure 25. Vector antenna element.

The components of the electric field are measured by use of the physical vibrators of the antenna element. We use the second Maxwell equation in integral form to calculate the components of the 
magnetic field vector (or rather, the vector collinear to vector $\vec{H}$, because we do not measure vector $\vec{E}$ directly, but the voltage on the vibrator loads $u$ associated with the field strength by the expression $\vec{E}=\overrightarrow{1_{0}} \cdot \frac{u}{h_{d}}$, where $h_{d}$ is the effective length of the vibrator and $\overrightarrow{1_{0}}$ is the unit vector, whose direction coincides with direction of the vector $\vec{E}$ ):

$$
\oint_{L} \vec{E} \overrightarrow{d l}=-\frac{d}{d t} \int_{S} \vec{B} \overrightarrow{d s}
$$

where $\overrightarrow{d l}$ is the unit vector element of the integration contour $L$, oriented counterclockwise; $\overrightarrow{d s}$ is the unit area element oriented normal to the surface $s$, based on the contour $L ; \vec{B}$ is the vector of magnetic induction penetrating the surface $s$.

The time derivative $-\frac{d}{d t}$ can be replaced by the factor $i \omega$; we substitute $\mu_{a} \vec{H}$ instead of $\vec{B}$; we also take into account that $\vec{E}=\overrightarrow{1_{0}} \cdot \frac{u}{h_{d}}, s=a^{2}$ (where $a$ is the side of the cube). By replacing the integration by summation, we can get following expression:

$$
H \cdot h_{d}=-\frac{\sum_{n=1}^{4} u_{n}^{(+,-)}}{i \omega \mu_{a} \cdot a},
$$

where $u_{n}^{(+,-)}$is the voltages at the vibrator loads.

Thus, due to the formation of VAA, it is possible to realize the measurement of the magnetic component of the electromagnetic field using exclusively electric vibrators.

Additionally, this vector antenna element can be used for estimating the angular coordinates of a radio source with arbitrary polarization and an arbitrary angle of incidence using the Poynting vector method. An elliptically polarized wave was used in the experiments, whose ratio of the horizontal axis to the vertical was 10; the azimuth of the incidence angle $(\varphi)$ was 30 degrees, and the elevation angle $(\theta)$ was 45 degrees.

The results of a numerical experiment of direction finding with a single vector antenna element using the Poynting vector method (first, the Poynting vector was calculated; then the real part of the Poynting vector was taken and its direction in space was determined, and as a result we obtained estimates of azimuth and elevation angle of the source) are presented at Figures 26 and 27.

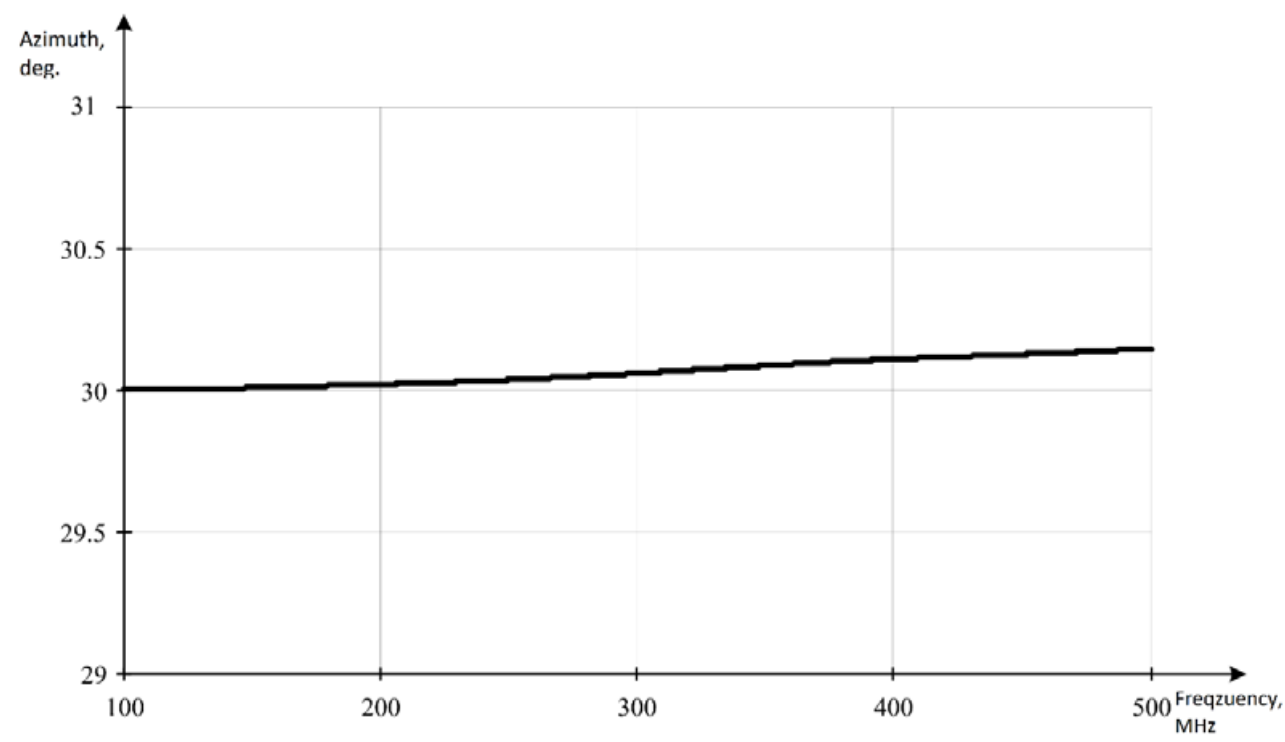

Figure 26. Calculated azimuth of the source in the frequency band from 100 to $500 \mathrm{MHz}$ (the true value of the azimuth is 30 degrees). 


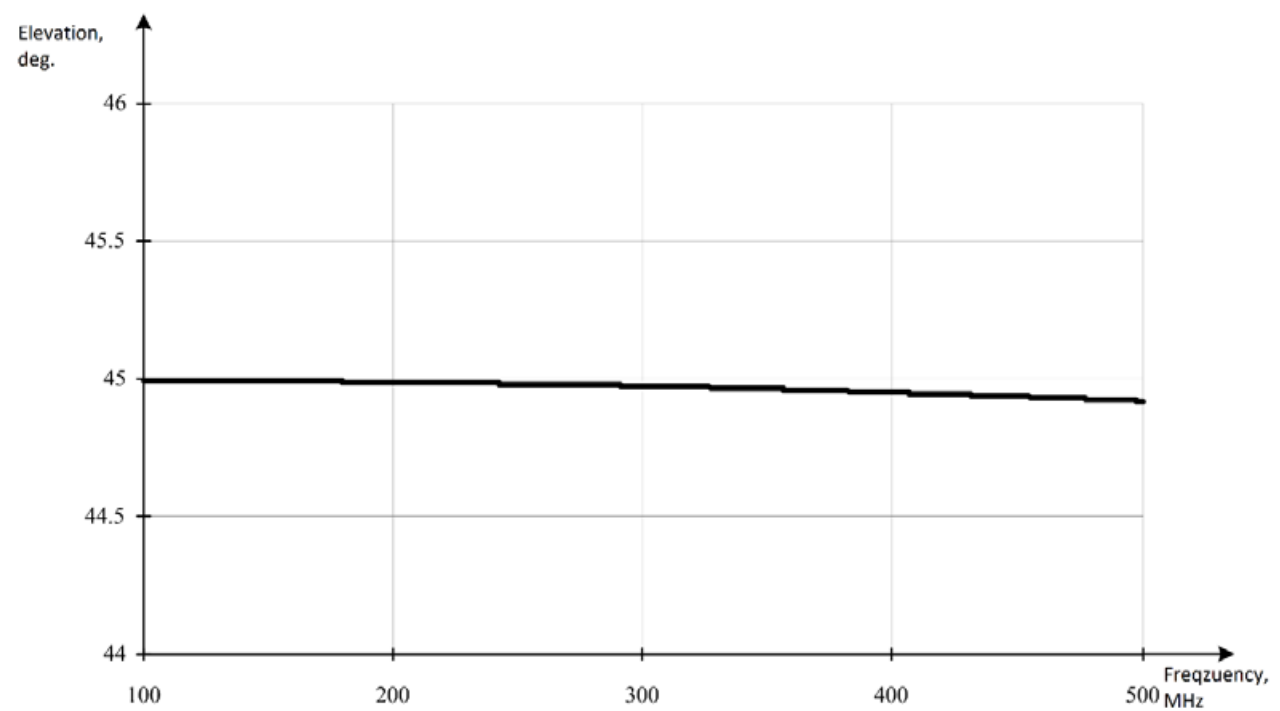

Figure 27. Calculated elevation angle of the source in the frequency band from 100 to $500 \mathrm{MHz}$ (the true elevation angle is 45 degrees).

Figures 26 and 27 show that the use of a single vector antenna element located in free space (Figure 25) can accurately measure the angular coordinates of the source by calculating the real part of the Poynting vector.

The considered model, where the direction-finding antenna array was located in free space, is idealized and allows us to give an initial assessment of the correctness of the proposed approach for the VAA formation. In real conditions, there are various scatterers in the immediate vicinity of the antenna array.

\section{Discussion and Conclusions}

Existing VAA methods are either based on the approximation of RF transparent array or implicitly contain information about the scattering properties of antenna array and its carrier. For example, they can use calibration procedure-waves incident on an antenna array from a number of directions, whereas we measure the signals at outputs of array elements and obtain the radiation patterns of array elements taking into account the influence of array and its carrier.

The problem is that an expensive and time-consuming calibration procedure assumes the geometry of direction finder body is sufficiently known. In particular: the doors and roof hatches are supposed to be closed; various direction finder elements have good electrical contact. During operation of direction finder, changes are inevitable: the presence of moisture and dirt unpredictably changes the resistance of contacting surfaces of metal sheets connected with rivets; opening doors and roof hatches during DOA estimation can also lead to a significant change in the currents flowing through the carrier body. Thus, after a short time of direction finder operation, the calibration performed by manufacturer becomes inadequate to the new electrodynamic properties of the carrier body.

Proposed VAA methods can reduce a DOA estimation error regardless of scatterer geometry and do not require a priori knowledge of its characteristics (geometry, material properties, or the scattering radiation patterns). We do not need to train a neural network that approximates the received antenna array signals depending on frequency and direction of wave incidence. With a random change in the geometry and material properties of the carrier, the proposed methods will still reduce DOA estimation error.

The VAA methods proposed in this article can increase the number of degrees of freedom of the receiving antenna system, which includes a physically existing (real) antenna array and additional spatial field samples (VAA). 
This allows one to significantly increase the number of deep zeros of the radiation pattern of the receiving antenna system (consisted of real and virtual antenna elements), and significantly weaken the sensitivity of the antenna system in the directions of interfering signals caused by wave reflections from surrounding objects [37]. Thus, the proposed methods for the VAA formation can be used to increase the accuracy of GNSS positioning.

There are some limitations of the proposed methods: the dimensions of the formed VAA cannot exceed the size of RAA by more than 2-4 times; for the number of elements of a RAA from seven to nine (this number of elements is often used in direction finders), the error in measuring the amplitude of the signals should not exceed $0.5-1 \mathrm{~dB}$, and the error in measuring the phase should be $5-10$ degrees.

Author Contributions: Conceptualization, Y.G.P. and A.V.A.; methodology, Y.G.P. and Y.A.R.; validation, S.M.F., A.V.A., and Y.A.R.; formal analysis, S.M.F.; investigation, S.M.F.; resources, A.V.A. and Y.A.R.; data curation, Y.G.P.; writing - original draft preparation, D.V.Z.; visualization, D.V.Z. All authors have read and agreed to the published version of the manuscript.

Funding: This research was funded by Russian Science Foundation, grant number 19-79-10109.

Acknowledgments: The authors would like to thank IRCOS JSC (Moscow) for provided research equipment.

Conflicts of Interest: The authors declare no conflict of interest.

\section{References}

1. Friedlander, B. Direction finding with an interpolated array. In Proceedings of the International Conference on Acoustics, Speech, and Signal Processing, Albuquerque, NM, USA, 3-6 April 1990; pp. 2951-2954.

2. Tuncer, E.; Friedlander, B. Classical and Modern Direction-of-Arrival Estimation; Academic Press: New York, NY, USA, 2009; p. 456.

3. Pesavento, M.; Gershman, A.B.; Luo, Z.-Q. Robust array interpolation using second-order cone programming. IEEE Signal Process. Lett. 2002, 9, 8-11. [CrossRef]

4. Buhren, M.; Pesavento, M.; Bohme, J.F. A new approach to array interpolation by generation of artificial shift invariances: Interpolated ESPRIT. In Proceedings of the 2003 IEEE International Conference on Acoustics, Speech, and Signal Processing, Hong Kong, China, 6-10 April 2003; Volume 5, pp. 205-208.

5. Hyberg, P. Antenna Array Mapping for DOA Estimation in Radio Signal Reconnaissance. Ph.D. Thesis, Royal Institute of Technology (KTH), Stockholm, Sweden, 2005.

6. Belloni, F.; Richter, A.; Koivunen, V. Reducing excess variance in beamspace methods for uniform circular array. In Proceedings of the IEEE Workshop on Statistical Signal Processing (SSP), Bordeaux, France, 17-20 July 2005; pp. 940-943.

7. Chen, S.; Mulgrew, B.; Grant, P.M. A clustering technique for digital communications channel equalization using radial basis function networks. IEEE Trans. Neural Netw. 1993, 4, 570-590. [CrossRef] [PubMed]

8. Belloni, F.; Richter, A.; Koivunen, V. DOA estimation via manifold separation for arbitrary array structures. IEEE Trans. Signal Process. 2007, 55, 4800-4810. [CrossRef]

9. Chandran, S. Advances in Direction-of-Arrival Estimation; Artech House: Norwood, MA, USA, 2006; p. 496.

10. van Rooyen, P.; Roux, P. Antenna Array Including Virtual Antenna. Patent US 7,605,755B2, 20 October 2009.

11. Salonen, I.; Vainikainen, P. Optimal virtual element patterns for adaptive arrays. IEEE Trans. Antennas Propag. 2016, 54, 204-210. [CrossRef]

12. Ahmed, I.; Perger, W. Direction finding in the presence of near zone resonant size scatterers. Prog. Electromagn. Res. B 2013, 56, 219-234. [CrossRef]

13. Gupta, P.; Agrawal, M. Design and analysis of the sparse array for DoA estimation of noncircular signals. IEEE Trans. Signal Process. 2019, 67, 460-473. [CrossRef]

14. Wang, X.; Xiong, Y.; Huang, W. An accurate direction finding scheme using virtual antenna array via smartphones. Sensors 2016, 16, 1811. [CrossRef]

15. Nguyen, H.; Mahler, K.; Peter, M.; Keusgen, W.; Eichler, T.; Mellein, H. Estimation of DoA based on large-scale virtual array data. In Proceedings of the 2016 10th European Conference on Antennas and Propagation (EuCAP), Davos, Switzerland, 10-15 April 2016; pp. 1-4. 
16. Zhou, C.; Shi, Z.; Gu, Y.; Zhang, Y.D. Coarray interpolation-based coprime array DoA estimation via covariance matrix reconstruction. In Proceedings of the 2018 IEEE International Conference on Acoustics, Speech and Signal Processing (ICASSP), Calgary, AB, Canada, 15-20 April 2018; pp. 3479-3483.

17. Lin, M.; Cao, L.; Ouyang, J.; Shi, W.; An, K. DOA estimation using virtual array technique for noncirlular signals. In Proceedings of the 2012 International Conference on Wireless Communications and Signal Processing (WCSP), Huangshan, China, 25-27 October 2012; pp. 1-5.

18. Liu, C.-L.; Vaidyanathan, P.P.; Pal, P. Coprime coarray interpolation for DoA estimation via nuclear norm minimization. In Proceedings of the 2016 IEEE International Symposium on Circuits and Systems (ISCAS), Montreal, QC, Canada, 22-25 May 2016; pp. 2639-2642.

19. Mahmud, T.H.A.; Ye, Z.; Shabir, K.; Zheng, R.; Islam, M.S. Off-grid DOA estimation aiding virtual extension of coprime arrays exploiting fourth order difference co-array with interpolation. IEEE Access 2018, 6, 46097-46109. [CrossRef]

20. Lee, S.; Kim, S.-C. Logarithmic-domain array interpolation for improved direction of arrival estimation in automotive radars. Sensors 2019, 19, 2410. [CrossRef]

21. Jackson, J.D. Classical Electrodynamics; Wiley: New York, NY, USA, 1998; p. 832.

22. Korn, G.A.; Korn, T.M. Mathematical Handbook for Scientists and Engineers: Definitions, Theorems, and Formulas for Reference and Review; Dover Publications: New York, NY, USA, 2000; p. 1152.

23. Bakhvalov, Y.A.; Knyazev, S.Y.U.; Shcherbakov, A.A. Mathematical modeling of physical fields by the point-source method. Bull. Russ. Acad. Sci. Phys. 2008, 72, 1190-1192. [CrossRef]

24. Levenberg, K. A method for the solution of certain non-linear problems in least squares. Quart. Appl. Math. 1944, 2, 164-168. [CrossRef]

25. Ashikhmin, A.V.; Pasternak, Y.G.; Podvalny, S.L.; Rembovsky, Y.A. Application of cauchy integral to approximate the field components and current in metal \& dielectrical polygons-Method of the field (current) restoration inside and outside flat closed contour based on its known values at the contour. In Proceedings of the International Conference on Wireless Information Networks and Systems (WINSYS), Porto, Portugal, 26-29 July 2008; pp. 153-156.

26. Rembovskii, Y.A.; Ivanov, A.V.; Pasternak, Y.G. Study of the method for compensation of systematic errors in direction-finding of radio emission sources that is based on cauchy integral properties. Telecommun. Radio Eng. 2009, 68, 1643-1650. [CrossRef]

27. Ashikhmin, A.V.; Negrobov, V.V.; Pasternak, Y.G.; Rembovskii, Y.A. Investigation of the possibility for description of the spatial field distribution near a mobile radio direction finder antenna system by a laurent series. Telecommun. Radio Eng. 2012, 71, 803-816. [CrossRef]

28. Ashikhmin, A.V.; Pasternak, Y.G.; Rembovsky, Y.A.; Fedorov, S.M. Study of field distortions introduced by antenna array mobile carrier body. Microw. Opt. Technol. Lett. 2018, 60, 2250-2252. [CrossRef]

29. Rembovsky, A.; Ashikhmin, A.; Kozmin, V.; Smolskiy, S. Radio Monitoring. Problems, Methods, and Equipment; Springer: New York, NY, USA, 2009; p. 507.

30. Ge, Y.; Dai, P.; Qin, W.; Yang, X.; Zhou, F.; Wang, S.; Zhao, X. Performance of multi-GNSS precise point positioning time and frequency transfer with clock modeling. Remote Sens. 2019, 11, 347. [CrossRef]

31. Maciuk, K. GPS-only, GLONASS-only and combined GPS+GLONASS absolute positioning under different sky view conditions. Teh. Vjesn. Tech. Gaz. 2018, 25, 933-939.

32. Wang, G. Millimeter-accuracy GPS landslide monitoring using Precise Point Positioning with Single Receiver Phase Ambiguity (PPP-SRPA) resolution: A case study in Puerto Rico. J. Geod. Soc. 2013, 3, 22-31. [CrossRef]

33. Rabbou, M.; El-Rabbany, A. PPP accuracy enhancement using GPS/GLONASS observations in kinematic mode. Positioning 2015, 6, 1-6. [CrossRef]

34. Wu, Q.; Sun, M.; Zhou, C.; Zhang, P. Precise point positioning using dual-frequency GNSS observations on smartphone. Sensors 2019, 19, 2189. [CrossRef]

35. Zhang, B.; Ou, J.; Yuan, Y.; Li, Z. Extraction of line-of-sight ionospheric observables from GPS data using precise point positioning. Sci. China Earth Sci. 2012, 55, 1919-1928. [CrossRef] 
36. Khalatbari, S.; Sardari, D.; Mirzaee, A.A.; Sadafi, H.A. Calculating SAR in two models of the human head exposed to mobile phones radiations at 900 and 1800 MHz. Prog. Electromagn. Res. Symp. 2006, 2, 104-109. [CrossRef]

37. Kos, T.; Markezic, I.; Pokrajcic, J. Effects of multipath reception on GPS positioning performance. In Proceedings of the ELMAR-2010, Zadar, Croatia, 15-17 September 2010; pp. 399-402.

(C) 2020 by the authors. Licensee MDPI, Basel, Switzerland. This article is an open access article distributed under the terms and conditions of the Creative Commons Attribution (CC BY) license (http://creativecommons.org/licenses/by/4.0/). 